\title{
A Family of Directional Relation Models for Extended Objects
}

\author{
Spiros Skiadopoulos, Nikos Sarkas, Timos Sellis, Member, IEEE, and \\ Manolis Koubarakis, Member, IEEE Computer Society
}

\begin{abstract}
In this paper, we introduce a family of expressive models for qualitative spatial reasoning with directions. The proposed family is based on the cognitive plausible cone-based model. We formally define the directional relations that can be expressed in each model of the family. Then, we use our formal framework to study two interesting problems: computing the inverse of a directional relation and composing two directional relations. For the composition operator, in particular, we concentrate on two commonly used definitions, namely, consistency-based and existential composition. Our formal framework allows us to prove that our solutions are correct. The presented solutions are handled in a uniform manner and apply to all of the models of the family.
\end{abstract}

Index Terms—Spatial databases and GIS, cone-based directional relations, inverse and composition operators.

\section{INTRODUCTION}

$\mathrm{T}$ HE subject of this paper belongs to the broader research area of Qualitative Spatial Reasoning (QSR). The goal of QSR is to approach common sense knowledge and reasoning about space by using symbolic and not numerical methods. It is no surprise that QSR has found applications in many diverse scientific areas that concentrate on building successful intelligent systems: geographic information systems (GISs) [1], [2], artificial intelligence [3], [4], databases [5], [6], and multimedia [7], just to name a few. Most researchers have concentrated on the three main aspects of space, namely, topology [8], [3], [1], [4], distance [2], [9], and orientation [10], [11], [12], [13], [14], [15], [16], [17]. The uttermost aim in these lines of research is to define new categories of spatial operators, as well as to build efficient algorithms for the automatic processing of queries using such operators.

In this paper, we consider extended objects and concentrate on binary directional relations. Such relations describe how a primary object $a$ is placed relative to a reference object $b$ by utilizing a coordinate system (for example, object $a$ is north of object $b$ ). Early qualitative models for directional relations approximate an extended spatial object by a representative point (most commonly, the centroid) [10],

- S. Skiadopoulos is with the Department of Computer Science and Technology, University of Peloponnese, Karaiskaki Street, 22100, Tripoli, Hellas. E-mail: spiros@uop.gr.

- N. Sarkas is with the Department of Computer Science, University of Toronto, 10 King's College Road, M5S 3G4, Toronto, ON, Canada. E-mail: nsarkas@cs.toronto.edu.

- T. Sellis is with the School of Electrical and Computer Engineering, Computer Science Division, National Technical University of Athens, 9, Iroon Polytechneiou Street, GR-15773 Zographou, Athens, Hellas. E-mail: timos@dblab.ece.ntua.gr.

- M. Koubarakis is with the Department of Informatics and Telecommunications, National and Kapodistrian University of Athens, Panepistimiopolis, Ilisia, Athens 15784, Greece. E-mail: koubarak@di.uoa.gr.

Manuscript received 16 Feb. 2006; revised 13 Jan. 2007; accepted 30 Mar. 2007; published online 18 Apr. 2007.

For information on obtaining reprints of this article, please send e-mail to tkde@computer.org, and reference IEEECS Log Number TKDE-0081-0206.

Digital Object Identifier no. 10.1109/TKDE.2007.1046.

$1041-4347 / 07 / \$ 25.00$ (C) 2007 IEEE
[2], [12], [16]. Typically, such models partition the space around the reference object $b$ into a number of mutually exclusive areas. For instance, the projection-based model partitions the space by using lines parallel to the axes (Fig. 1a), whereas the cone-based model partitions the space by using lines with an origin angle $\phi$ (Fig. 1b). Depending on the adopted model, the relation between two objects may change. For instance, consider Fig. 1: According to the projection-based model, $a$ is northeast of $b$ (Fig. 1a), whereas, according to the cone-based model, $a$ is north of $b$ (Fig. 1b). Later models approximate an object by a representative area (most commonly, the minimum bounding box (mbb)) and express directions on these approximations [14], [15]. Unfortunately, models that approximate both the primary and the reference objects may give misleading directional relations when objects are overlapping, intertwined, or horseshoe shaped (for an extended discussion, see [18]).

More recently, Goyal [18] and Skiadopoulos and Koubarakis [17], [19] studied a model that expresses the directional relation by only approximating the reference object $b$ (using its mbb) while using the exact shape of the primary object $a$. Intuitively, this model 1) partitions the plane around the reference object into nine areas similar to the projection-based model (these areas correspond to directional relations such as north, northeast, and so forth) and 2) records the areas occupied by the primary object. These areas provide the directional relation between the primary and the reference objects. For instance, in Fig. 1c, object $a$ is partly $N E$ and partly $E$ of object $b$. We denote this model by Projection-based Directional Relations $(\mathcal{P} D R)$. Clearly, the $\mathcal{P} D R$ model offers a more precise and expressive model than previous approaches that approximate objects by using points or boxes [18].

However, the $\mathcal{P} D R$ model is not without weaknesses. The number of relations that can be expressed in the model is very large (511 relations). Furthermore, the $\mathcal{P} D R$ model partitions the reference space similar to the projection-based model by using lines parallel to the axes (Fig. 1c). Most people do not find this partition natural. 


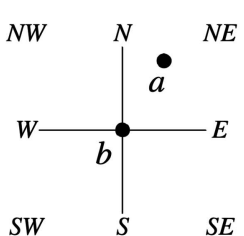

(a)

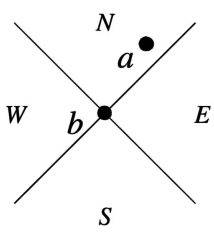

(b)

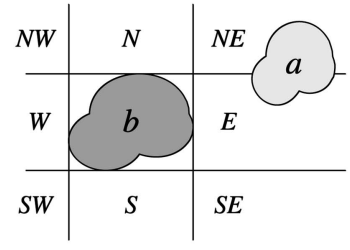

(c)
Fig. 1. Models of directional relations.

Typically, people tend to organize surrounding space by using lines with an origin angle similar to the cone-based model (Fig. 1b). Hence, most people find the cone-based partition more intuitive and descriptive. The cognitive plausibility of the cone-based model has been verified by studies in the field of cognitive sciences (see, for instance, [20], [21]). Moreover, the cone-based partition is a typical approximation for the field of view of the human eye and camera lenses [22], [23]. For the above reasons, cone-based models have been used in computer vision [22], [24], robot navigation [25], and GISs [11], [26].

In this paper, we propose Cone-based Directional Relations $(C D R)$, an alternative family of directional relation models that is based on the cognitive plausible cone-based model. In the $\mathcal{C} D R$ family of models, only the reference object is approximated by its $\mathrm{mbb}$ (as in $\mathcal{P} D R$ ), but the space around the reference object is partitioned into five areas by using the cone-based model. The family contains an infinite number of models. Each model in the $\mathcal{C} D R$ family is identified by a unique value for $\phi\left(0^{\circ}<\phi<90^{\circ}\right)$ that defines the origin angle of the space partitioning lines (see also Fig. 2a). In other words, for each particular application, by choosing a suitable value for $\phi$, we can find an appropriate model in the $\mathcal{C} D R$ family. Moreover, $\mathcal{C} D R$ models result in a set of 31 relations-a significantly smaller set compared to $\mathcal{P} D R$, which has 511 relations.

We formally define the relations that can be expressed in the $\mathcal{C} D R$ family and focus on two interesting problems: computing the inverse of a directional relation and composing two directional relations. The inverse and composition operations for various kinds of spatial relations have received considerable attention in the literature [27], [1], [2], [15], [4], [12]. For the composition operator, in particular, research has mainly concentrated on two definitions, namely, existential and consistency-based composition [8], [28]. Existential composition is the standard definition of composition from the set theory [8], [28], [15], [4]. Consistency-based composition is a weaker interpretation useful in several domains [29], [30].

The inverse and composition operations are used as mechanisms for inferring new spatial relations from existing ones. Such mechanisms are important as they are at the heart of any system that retrieves collections of objects similarly related to each other by using spatial relations. For instance, these inference mechanisms are very helpful when we need to detect inconsistencies in a given set of spatial relations [16], [4] or preprocess spatial queries and prune the search space [31]. Inverse and composition are also essential parts of Relation Algebras [32], [33], [34], so their formal study is a prerequisite to any algebraic approach to

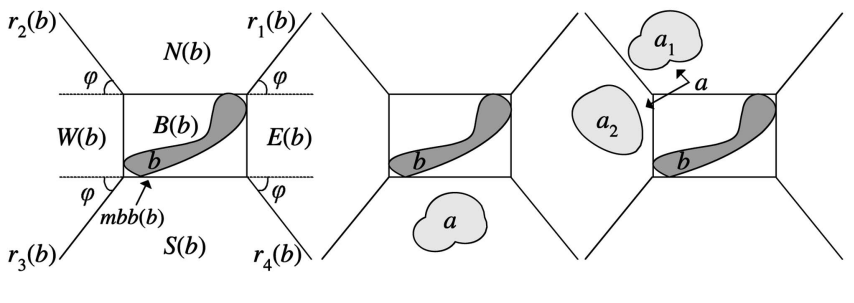

(a)

(b)

(c)

Fig. 2. Reference tiles and relations.

spatial reasoning. Moreover, composition is often used to identify classes of relations that have a tractable consistency problem [27], [4], [35].

The technical contributions of this paper can be summarized as follows:

- We propose the $\mathcal{C} D R$ family of directional relation models. The relations that can be expressed in each model of the family are formally defined. $\mathcal{C} D R$ models are based on the cognitive plausible conebased model and can be customized to serve a wide variety of applications. Finally, $\mathcal{C} D R$ offers a small and easy-to-manage set of relations.

- We consider the inverse operation for directional relations in the $\mathcal{C} D R$ family. We present a method to compute the inverse of a relation and formally prove its correctness.

- We study the problem of composing two directional relations of the $\mathcal{C} D R$ family. We first present a method for consistency-based composition. To this end, we consider progressively more expressive classes of directional relations and present consistency-based composition algorithms for these classes. Our theoretical framework allows us to prove formally that our algorithms are correct. Finally, we consider the existential definition of composition. Contrary to consistency-based composition, we show that the binary relation resulting from the existential composition of two directional relations cannot always be expressed using the relations of the $\mathcal{C} D R$ family.

The rest of the paper is organized as follows: Section 2 defines the $\mathcal{C} D R$ family of directional relation models. In Section 3, we study the inverse and composition problem for the directional relations in the $\mathcal{C} D R$ family. Finally, Section 4 offers conclusions and discusses future research directions.

\section{A family of Directional Relation Models}

In this section, we present the $\mathcal{C} D R$ family of directional relation models. We consider the euclidean space $\Re^{2}$. Objects are defined as nonempty and bounded sets of points in $\Re^{2}$. Let $a$ be an object. The $m b b$ of object $a$, denoted by $m b b(a)$, is the smallest rectangle, aligned with the axes, that encloses the object (Fig. 2a). Throughout this paper, we will consider objects that are formed by finite unions of objects that are homeomorphic to the closed unit disk [17]. This set of objects is denoted by $R E G^{*}$. Objects in $R E G^{*}$ can be disconnected and have holes. However, class $R E G^{*}$ 
excludes points, lines, and objects with emanating lines. A thorough discussion about $R E G^{*}$ and the way objects are modeled in $R E G^{*}$ appears in [36], [19].

To define a relation in $\mathcal{C} D R$ between a primary object $a$ and a reference object $b$, we consider the mbb of object $b$ and four rays originating from its four vertices. We denote by $r_{1}(b)$ (respectively, $r_{2}(b), r_{3}(b)$, and $r_{4}(b)$ ) the ray that originates from the upper right (respectively, the upper left, lower left, and lower right) vertex of $m b b(b)$ (see also Fig. 2a). The origin angle of each ray is presented in Fig. 2a. Note that the origin angle has the same value for all rays such that the plane is partitioned symmetrically. This angle, denoted by $\phi$, is called the characteristic angle of the model and can have values in the interval $\left(0^{\circ}, 90^{\circ}\right){ }^{1}$

Every possible value of $\phi$ specifies a new model in the $\mathcal{C} D R$ family. Such a model will be denoted by $\mathcal{C} D R(\phi)$. For instance, $\mathcal{C} D R\left(30^{\circ}\right)$ denotes the model of $\mathcal{C} D R$, where $\phi=30^{\circ}$. Notice that the value of $\phi$ is fixed within a certain model $\mathcal{C} D R(\phi)$ and is not allowed to vary for different objects. The analysis that we present in this paper is valid for any model $\mathcal{C} D R(\phi)$ in the $\mathcal{C} D R$ family $\left(0^{\circ}<\phi<90^{\circ}\right)$. Wherever necessary, in the material that follows, the value of $\phi$ appears as a parameter.

The mbb of the reference object $b$, along with the four rays, divides the plane into five areas, which we call tiles (Fig. 2a). The peripheral tiles correspond to the four directional relations north, west, south, and east. These tiles will be denoted by $N(b), W(b), S(b)$, and $E(b)$, respectively. The central area corresponds to the object's mbb and is denoted by $B(b)$. Notice that

1. all tiles are closed,

2. all tiles but $B(b)$ are unbounded,

3. the union of all five tiles is $\Re^{2}$, and

4. two distinct tiles have disjoint interiors but may share points in their boundaries (for instance, $W(b)$ and $B(b)$ share the left side of the mbb of $b$ ).

Even though tiles share some points along their borders, there is no ambiguity in defining relations in the $\mathcal{C} D R$ family because class $R E G^{*}$ does not contain objects that could lie entirely on the borderline (like lines, points, and objects with emanating lines).

Informally, if a primary object $a$ is included (in the settheoretic sense) in tile $S(b)$ of some reference object $b$ (Fig. 2b), then we say that $a$ is south of $b$ and we write $a S b$. Similarly, we can define north $(N)$, west $(W)$, east $(E)$, and bounding box $(B)$ relations. If a primary object $a$ lies partly in tile $N(b)$ and partly in tile $W(b)$ of some reference object $b$ (Fig. 2c), then we say that $a$ is partly north and partly west of $b$ and we write $a N: W b$.

The general definition of a basic directional relation in our framework is given as follows:.

Definition 1. A basic directional relation is an expression $R_{1}: \cdots: R_{k}$, where

1. $1 \leq k \leq 5$,

2. $R_{1}, \ldots, R_{k} \in\{N, W, S, E, B\}$, and

3. $R_{i} \neq R_{j}$ for every $i$, $j$ such that $1 \leq i, j \leq k$, and $i \neq j$.

1. For values $\phi=0^{\circ}$ and $\phi=90^{\circ}$, the model degenerates to a subcase of $\mathcal{P} D R$.
A basic directional relation $R_{1}: \cdots: R_{k}$ is called single tile if $k=1$; otherwise, it is called multitile.

Example 1. Expressions $S$ and $N: W$ are basic directional relations. The first is a single-tile relation, whereas the second is a multitile. Objects involved in these relations are shown in Figs. $2 b$ and $2 c$, respectively.

In order to avoid confusion, we will write the single-tile elements of a basic directional relation according to the following order: $N, W, S, E$, and $B$. Thus, we always write $N: W: B$ instead of $W: B: N$ or $N: B: W$. Moreover, for a relation such as $N: W: B$, we will often refer to $N, W$, and $B$ as its tiles.

The set of basic directional relations (single or multitile) in every $\mathcal{C} D R$ model contains $\sum_{i=1}^{5}\left(\begin{array}{l}5 \\ i\end{array}\right)=31$ elements. We will use $\mathcal{B}^{*}$ to denote this set. Relations in $\mathcal{B}^{*}$ are jointly exhaustive and pairwise disjoint and can be used to represent definite information about directions. Thus, relations in $\mathcal{B}^{*}$ express precise knowledge like object $a$ is north of $b$, denoted by a $N$ b. Using the relations of $\mathcal{B}^{*}$ as our basis, we can define the power set $2^{\mathcal{B}^{*}}$ of $\mathcal{B}^{*}$, which contains $2^{31}$ relations. Elements of $2^{\mathcal{B}^{*}}$ are called directional relations and can be used to represent not only definite but also indefinite information about directions. Thus, relations in $2^{\mathcal{B}^{*}}$ also express imprecise knowledge like object $a$ is either partly north and partly west or entirely west of object $b$, denoted by $a\{N: W, W\}$ b. In general, expression $a \cup_{i=1}^{n} R_{i} b$ denotes that object $a$ is related to $b$, with some relation among $R_{1}, \ldots, R_{n}$. We will use $Q, Q_{1}, Q_{2}, \ldots$ to denote directional relations and $R, R_{1}, R_{2}, \ldots$ to denote basic directional relations, either single tile or multitile.

Let us now highlight the advantages of the proposed model.

Cognitive plausibility. $\mathcal{C} D R$ models are based on the cone-based partition of space that is close to the human perception of direction, as shown by cognitive science studies [20], [21]. Informally, the cone-based partition is a typical approximation for the field of view of camera lenses and the human eye [22], [23]. Cone-based models have been used in computer vision [22], [24], robot navigation [25], and GISs [11], [26].

Applicability. The $\mathcal{C} D R$ models can be used in a wide set of applications that use directions like GISs and robot navigation. In this paper, we have focused on the GISs paradigm and use cardinal direction relations (like West). The model can also be used in other applications by simply renaming the appropriate relations (for instance, using Left instead of West). For completeness, let us also give a robot navigation example [25]. Consider a set of robots, equipped with perceptual capabilities, exploring an unknown area. This process can be optimized if robots move toward unexplored areas. Thus, every robot should know the position and the explored area of every other robot. To this end, the robots could compose and broadcast a complete metric map of their vicinity. This solution is costly mainly because metric maps are hard to compute and broadcast. Alternatively, every robot could 1) locate the landmarks and the robots in its vicinity, 2) identify their relations (using the $\mathcal{C} D R$ model), and 3) broadcast this information to the other robots. This qualitative solution does not require full metric mapping capabilities, requires significantly 


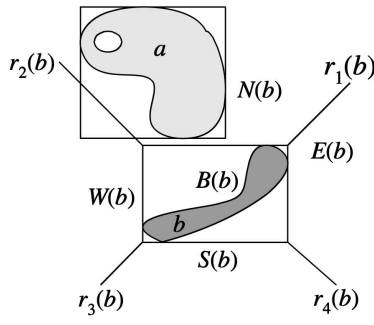

(a)

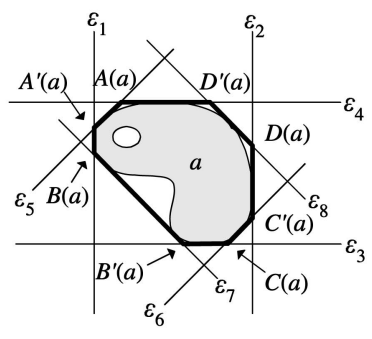

(b)
Fig. 3. The minimum bounding octagon.

smaller bandwidth, and typically is sufficient for the exploring task. Similarly, robots can use $\mathcal{C} D R$ relations to retain their formation.

Customization. The $\mathcal{C} D R$ models are distinguished according to the parameter $\phi\left(0^{\circ}<\phi<90^{\circ}\right)$ that defines the origin angle of the space separating lines (Fig. 2a). For each particular application, by choosing a suitable $\phi$, we can find an appropriate model in the $\mathcal{C} D R$ family. For instance, in a robot navigation application where the field of view angle of the lenses used in the robot's vision system is $30^{\circ}$, we may choose to use the $\mathcal{C} D R\left(30^{\circ}\right)$ model.

Small set of relations. The $\mathcal{C} D R$ models can express a small and easy-to-use set of 31 jointly exhaustive and pairwise disjoint relations. This number is significantly smaller than the respective set of $\mathcal{P} D R$ relations, which contains 511 relations.

The next section formally defines the relations that can be expressed in the $\mathcal{C} D R$ family.

\subsection{Defining Directional Relations Formally}

Intuitively, in order to derive the basic directional relation between a primary object $a$ and a reference object $b$, one needs to identify the tiles of the plane induced by $b$, where object $a$ lies. However, such an intuitive (but informal) definition is generally inadequate for the study of a spatial model.

For the $\mathcal{P} D R$ model, two objects are related through a single-tile directional relation iff their mbbs are related with the same relation. This observation allows the definition of single-tile relations using sets of conditions involving the vertex coordinates of the objects' mbbs [17]. This more elaborate definition was subsequently used to study the inverse, composition, and consistency checking problems for that particular model.

We will attempt to derive such a definition for the proposed family of models. We can easily demonstrate that the above observation does not hold for the proposed $\mathcal{C} D R$ family. For instance, in Fig. 3a, notice that, whereas $a N b$, we have $m b b(a) N: W b$. Thus, the mbb provides a crude approximation of an object in the $\mathcal{C} D R$ family. However, a more precise approximation exists and can be constructed as follows:

Let us consider an arbitrary model $\mathcal{C} D R(\phi)$. We further refine the $\mathrm{mbb}$ of an object by using lines parallel to rays $r_{1}, \ldots, r_{4}$. After we form the mbb around the object, we also form four lines, tangent to the object and parallel to the rays. We use those lines to clip the corners of the mbb. By doing so, we come up with a new approximation, which we call

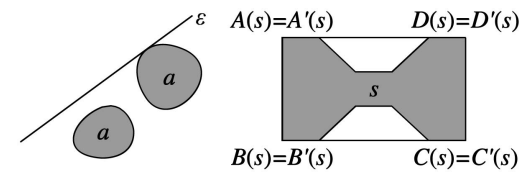

(a)

(b)

Fig. 4. (a) Tangency and (b) a degenerated minimum bounding octagon.

mbo (Fig. 3b). The mbo is formally defined in Definition 2 and we will later see that it is appropriate for defining relations in $\mathcal{C} D R(\phi)$. Notice that the mbo is a special eightcorner approximation belonging to the general class of minimum bounding $n$-corner approximations for $n=8$ [37].

Notation 1. We denote by

- $O_{x}$ (respectively, $O_{y}$ ) the $x$-coordinate (respectively, the $y$-coordinate) of a point $O$,

- $\varepsilon_{1} \mid \varepsilon_{2}$ the intersection point of lines $\varepsilon_{1}$ and $\varepsilon_{2}$, and

- $(\varepsilon)_{x}$ (respectively, $(\varepsilon)_{y}$ ) the $x$-coordinate (respectively, the $y$-coordinate) of the intersection point of line $\varepsilon$ with the $x$-axis (respectively, the $y$-axis), that is, $(\varepsilon)_{x}=$ $(\varepsilon \mid x)_{x}$ and $(\varepsilon)_{y}=(\varepsilon \mid y)_{y}$.

Since set $R E G^{*}$ includes disconnected objects, we also need an appropriate definition of tangency. Thus, a line is tangent to an object in $R E G^{*}$ if it is tangent to one of its components, and the whole object lies on a single side of the line. For example, in Fig. $4 a$, line $\varepsilon$ is tangent to the composite object $a$.

Definition 2. Let $\mathcal{C} D R(\phi)$ be an arbitrary model in the $\mathcal{C} D R$ family (where $\phi$ is the characteristic angle of the model). The minimum bounding octagon (mbo) of an object $a \in R E G^{*}$, denoted by $m b o_{\phi}(a)$, is the polygon created by lines $\varepsilon_{1}, \ldots, \varepsilon_{8}$, where

1. $\varepsilon_{1}, \ldots, \varepsilon_{8}$ are tangential to $a$,

2. $\varepsilon_{1}, \varepsilon_{2}$ are parallel to the $y$-axis and $\left(\varepsilon_{1}\right)_{x}<\left(\varepsilon_{2}\right)_{x^{\prime}}$

3. $\varepsilon_{3}, \varepsilon_{4}$ are parallel to the $x$ axis and $\left(\varepsilon_{3}\right)_{y}<\left(\varepsilon_{4}\right)_{y^{\prime}}$

4. $\varepsilon_{5}, \varepsilon_{6}$ form angle $\phi$ with the $x$-axis and $\left(\varepsilon_{5}\right)_{x}<\left(\varepsilon_{6}\right)_{x^{\prime}}$ and

5. $\varepsilon_{7}, \varepsilon_{8}$ form angle 180 degrees $-\phi$ with the $x$-axis, and $\left(\varepsilon_{7}\right)_{x}<\left(\varepsilon_{8}\right)_{x}$.

The points forming the polygon presented in a counterclockwise order are: $A(a)=\varepsilon_{4}\left|\varepsilon_{5}, \quad A^{\prime}(a)=\varepsilon_{1}\right| \varepsilon_{5}$, $B(a)=\varepsilon_{1}\left|\varepsilon_{7}, \quad B^{\prime}(a)=\varepsilon_{3}\right| \varepsilon_{7}, \quad C(a)=\varepsilon_{3}\left|\varepsilon_{6}, \quad C^{\prime}(a)=\varepsilon_{2}\right| \varepsilon_{6}$, $D(a)=\varepsilon_{2} \mid \varepsilon_{8}$, and $D^{\prime}(a)=\varepsilon_{4} \mid \varepsilon_{8}$ (Fig. 3b).

Example 2. In certain cases, depending on the shape of object $a, m b o_{\phi}(a)$ can degenerate to a polygon having three, four, five, six, or seven vertices. For instance, in Fig. $4 \mathrm{~b}$, the mbo of object $s$ has only four vertices. This fact does not affect our analysis.

To define the mbo, we only need to specify four points (instead of eight). ${ }^{2}$ More specifically, vertices $A^{\prime}, B^{\prime}, C^{\prime}$, and $D^{\prime}$ can be computed using vertices $A, B, C$, and $D$ as follows:

2. Similarly to the mbb, where we need two points (instead of four). 


$$
\begin{array}{cc}
A_{x}^{\prime}=B_{x} & A_{y}^{\prime}=A_{y}-\tan \phi\left(A_{x}-B_{x}\right) \\
B_{x}^{\prime}=B_{x}+\frac{1}{\tan \phi}\left(B_{y}-C_{y}\right) & B_{y}^{\prime}=C_{y} \\
C_{x}^{\prime}=D_{x} & C_{y}^{\prime}=C_{y}+\tan \phi\left(D_{x}-C_{x}\right) \\
D_{x}^{\prime}=D_{x}-\frac{1}{\tan \phi}\left(A_{y}-D_{y}\right) & D_{y}^{\prime}=A_{y} .
\end{array}
$$

Note also that, from the mbo, we can easily compute the mbb. For instance, in Fig. 3, the $m b b(a)$ is the box (with its sides aligned with the axes) specified by points $\left(B_{x}(a), C_{y}(a)\right)$ and $\left(D_{x}(a), A_{y}(a)\right)$.

Using the mbo, we can formally define single-tile relations.

Definition 3. Let 1) $\mathcal{C} D R(\phi)$ be an arbitrary model in the $\mathcal{C} D R$ family $\left.\left(0^{\circ}<\phi<90^{\circ}\right), 2\right)$ a and $b$ be two objects in $R E G^{*}$, and 3) $A(a), B(a), C(a)$, and $D(a)$ and $A(b), B(b), C(b)$, and $D(b)$ the vertices of $m b o_{\phi}(a)$ and $m b o_{\phi}(b)$, respectively. Relations $N, W, S, E$, and $B$ are defined as follows:

$$
\begin{array}{lrl}
a N b \quad \text { iff } \quad & \tan (\phi) B_{x}(a)+B_{y}(a) \geq \tan (\phi) B_{x}(b)+ \\
& A_{y}(b), \tan (\phi) C_{x}(a)-C_{y}(a) \leq \\
& \tan (\phi) D_{x}(b)-A_{y}(b) \text { and } C_{y}(a) \geq A_{y}(b), \\
a W b \quad \text { iff } \quad & \tan (\phi) D_{x}(a)+D_{y}(a) \leq \tan (\phi) B_{x}(b)+ \\
& A_{y}(b), \tan (\phi) C_{x}(a)-C_{y}(a) \leq \\
& \tan (\phi) B_{x}(b)-C_{y}(b) \text { and } D_{x}(a) \leq B_{x}(b), \\
a S b \quad \text { iff } \quad & \tan (\phi) A_{x}(a)-A_{y}(a) \geq \tan (\phi) B_{x}(b)- \\
& C_{y}(b), \tan (\phi) D_{x}(a)+D_{y}(a) \leq \\
& \tan (\phi) D_{x}(b)+C_{y}(b) \text { and } A_{y}(a) \leq C_{y}(b), \\
a E b \quad \text { iff } \quad & \tan (\phi) A_{x}(a)-A_{y}(a) \geq \tan (\phi) D_{x}(b)- \\
& A_{y}(b), \tan (\phi) B_{x}(a)+B_{y}(a) \geq \\
& \tan (\phi) D_{x}(b)+C_{y}(b) \text { and } B_{x}(a) \geq D_{x}(b), \\
a B b \quad \text { iff } & C_{y}(a) \geq C_{y}(b), A_{y}(a) \leq A_{y}(b), B_{x}(a) \geq \\
& B_{x}(b) \text { and } D_{x}(a) \leq D_{x}(b) .
\end{array}
$$

A single-tile relation between a primary object $a$ and a reference object $b$ can also be defined using the mbo of $a\left(m b o_{\phi}(a)\right)$ and the mbb of $b(m b b(b))$. Notice that the above definition is essentially equivalent to Definition 3 since, as we have previously seen, $m b b(b)$ can be easily computed from $m b o_{\phi}(b)$. We have expressed Definition 3 by using only mbos for two reasons. First, using the same type of approximation for both objects $a$ and $b$ results in a more simple and uniform definition. More importantly, the mbo of the reference object $b\left(m b o_{\phi}(b)\right)$ will be more useful in subsequent computations. For instance, it is easy to verify that $m b b(b)$ can only be used to compute relations where $b$ acts as a reference object, whereas $m b o_{\phi}(b)$ can be used to compute any relation involving $b$ (regardless of whether $b$ acts as a primary or a reference object).

Multitile directional relations are defined as follows:

Definition 4. Let $a$ and $b$ be two objects in $R E G^{*}$ and $R=R_{1}$ : $\cdots: R_{k}$ a multitile directional relation. Then, a $R_{1}: \cdots: R_{k} b$ holds iff there exist objects $a_{1}, \ldots, a_{k} \in R E G^{*}$ such that $a_{1} R_{1} b, \ldots, a_{k} R_{k} b$, and $a=a_{1} \cup \cdots \cup a_{k}$.

Example 3. In Fig. 2c, we have $a N: W b$, since there exist objects $a_{1}$ and $a_{2}$ in $R E G^{*}$ such that $a_{1} N b, a_{2} W b$, and $a=a_{1} \cup a_{2}$.

To avoid overloading in Fig. 2, we have not illustrated $m b o_{\phi}(a), m b o_{\phi}\left(a_{1}\right)$, and $m b o_{\phi}\left(a_{2}\right)$. In the rest of the paper, we will also omit the mbo of the primary object whenever the relation can be easily seen. Finally, in Definition 4, notice that for every $i, j$ such that $1 \leq i, j \leq k$, and $i \neq j, a_{i}$ and $a_{j}$ have disjoint interiors but may share points in their boundaries.

In the following section, we will study the problems of computing the inverse of a directional relation and the composition of two directional relations. Our results are valid for every model $\mathcal{C} D R(\phi)$ in the $\mathcal{C} D R$ family.

\section{InVERSE AND Composition}

In this section, we will study the problem of computing the inverse and the composition of directional relations in the $\mathcal{C} D R$ family. We first present a method for computing the inverse of a $\mathcal{C} D R$ relation and then a method for composing two $\mathcal{C} D R$ relations. The presented solutions are handled in a uniform manner and apply to all of the $\mathcal{C} D R(\phi)$ models of the $\mathcal{C} D R$ family. Let us first define the inverse of a relation.

Definition 5. Let $Q$ be a directional relation in $2^{\mathcal{B}^{*}}$. The inverse of relation $Q$, denoted by $\operatorname{inv}(Q)$, is another directional relation, which satisfies the following: For arbitrary objects a, $b \in R E G^{*}, a$ inv $(Q) b$ holds iff $b Q$ a holds.

Two definitions of composition appear in the literature. The first one is the standard existential definition from the set theory [8], [4].

Definition 6. Let $Q_{1}$ and $Q_{2}$ be directional relations in $2^{\mathcal{B}^{*}}$. The existential composition of relations $Q_{1}$ and $Q_{2}$, denoted by $Q_{1} ; Q_{2}$, is another directional relation from $2^{\mathcal{B}^{*}}$ which satisfies the following. For arbitrary objects $a$ and $c, a Q_{1} ; Q_{2} c$ holds iff there exists an object $b$ such that $a Q_{1} b$ and $b Q_{2} c$ hold.

The second definition is explained as follows [8], [28]:

Definition 7. Let $Q_{1}$ and $Q_{2}$ be directional relations in $2^{\mathcal{B}^{*}}$. The consistency-based composition of relations $Q_{1}$ and $Q_{2}$, denoted by $Q_{1} \circ Q_{2}$, is another directional relation from $2^{\mathcal{B}^{*}}$ which satisfies the following: $Q_{1} \circ Q_{2}$ contains all relations $R \in \mathcal{B}^{*}$ such that there exist objects $a, b, c \in R E G^{*}$ such that $a Q_{1} b, b Q_{2} c$, and $a R c$ hold.

The consistency-based definition of composition is weaker than the existential definition. Observe that $R_{1} ; R_{2} \subseteq$ $R_{1} \circ R_{2}$ holds. The above definitions are important and have attracted the interest of many researchers since they can be used as a mechanism for inferring new information from existing ones [8], [28], [4].

In this section, we first present a method to compute the inverse of a $\mathcal{C} D R$ relation (Lemmas 1 and 2 and Theorem 1). Then, we study consistency-based composition. We consider progressively more expressive classes of directional relations and give consistency-based composition algorithms for these classes (Lemmas 3, 4, and 5 and Theorem 2). Finally, we consider the existential definition of composition and show that the binary relation resulting from the existential composition of some directional relations cannot be expressed using the $\mathcal{C} D R$ relations. Our theoretical framework allows us to prove formally that our solutions are correct.

As we discussed in Section 2.1, relations in the $\mathcal{C} D R$ family are defined using the mbo, whereas $\mathcal{P} D R$ relations are defined using the mbb. When handling the inverse and 
composition problems, this difference is crucial and renders inapplicable the $m b b$-based technique developed in [17] for the $\mathcal{P} D R$ model. This led us to develop a new mbo-based strategy for handling the inverse and composition problems for the $\mathcal{C} D R$ family.

During the study of the inverse and composition problems, we will use the informal inclusion-based definition of basic directional relations. The formal definition involving the mbos of objects is used implicitly. We note that the mbo-based definition is not without use, as 1) it is implicitly used and 2) it is an integral part of the framework that will be required for the further study of the proposed models (for example, for the study of the consistency checking and variable elimination problems).

Before we present our results, we introduce the necessary notation.

Notation 2. Let $R_{1}, \ldots, R_{k}$ be single-tile directional relations.

We denote by $\delta\left(R_{1}, \ldots, R_{k}\right)$ the disjunction of all basic directional relations that can be constructed by combining the single-tile relations $R_{1}, \ldots, R_{k}$. For instance, $\delta(N, W, B)$ stands for the following directional relation:

$$
\{N, W, B, N: W, N: B, W: B, N: W: B\} .
$$

Moreover, we define

$$
\begin{aligned}
& \delta\left(R_{1}: \cdots: R_{k}\right)=\delta\left(R_{1}, \ldots, R_{k}\right) \text { and } \\
& \delta\left(\delta\left(R_{11}, \ldots, R_{1 k_{1}}\right), \ldots, \delta\left(R_{m 1}, \ldots, R_{m k_{m}}\right)\right)= \\
& \delta\left(R_{11}, \ldots, R_{1 k_{1}}, \ldots, R_{m 1}, \ldots, R_{m k_{m}}\right) .
\end{aligned}
$$

We denote by $U_{\text {dir }}$ the universal directional relation, that is, $U_{\text {dir }}=\delta(N, W, S, E, B)$.

Notation 3. Let $R \in\{N, W, S, E\}$ be a single tile. We denote by $R^{\leftarrow}$ (respectively, $R^{\rightarrow}$ and $R^{\downarrow}$ ) the tile that we meet by moving counterclockwise (respectively, clockwise and diametrically) from tile $R$. Given a relation $R$, the expressions $R^{\leftarrow}, R^{\rightarrow}$, and $R \downarrow$ are defined in the following table:

\begin{tabular}{|l||c|c|c|c|}
\hline$R$ & $N$ & $W$ & $S$ & $E$ \\
\hline \hline$R^{\leftarrow}$ & $W$ & $S$ & $E$ & $N$ \\
\hline$R^{\rightarrow}$ & $E$ & $N$ & $W$ & $S$ \\
\hline$R^{\downarrow}$ & $S$ & $E$ & $N$ & $W$ \\
\hline
\end{tabular}

Notation 4. Let $R_{1}, \ldots, R_{k}$ be basic directional relations. The tile union of $R_{1}, \ldots, R_{k}$, denoted by tile-union $\left(R_{1}, \ldots, R_{k}\right)$, is the basic directional relation that consists of all the tiles in relations $R_{1}, \ldots, R_{k}$. Furthermore, we denote by Combine $\left(Q_{1}, \ldots, Q_{k}\right)$ (where $Q_{1}, \ldots, Q_{k} \in 2^{\mathcal{B}^{*}}$ ) the directional relation

$$
\begin{aligned}
\left\{R \in \mathcal{B}^{*}: R\right. & =\operatorname{tile-union}\left(s_{1}, \ldots, s_{k}\right) \\
& \left.\wedge s_{1} \in Q_{1} \wedge \cdots \wedge s_{k} \in Q_{k}\right\} .
\end{aligned}
$$

Example 4. Consider two basic directional relations: $N: W$ and $N: E: B$. Then,

$$
\text { tile-union }(N: W, N: E: B)=N: W: E: B .
$$

Furthermore, consider two directional relations: $\{N, N$ : $W\}$ and $\{S, S: E\}$. Then,

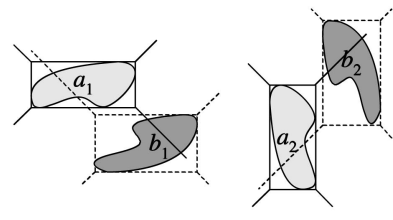

(a) (b)

Fig. 5. Proving Proposition 1.

$$
\begin{aligned}
& \text { Combine }(\{N, N: W\},\{S, S: E\})= \\
& \left\{\begin{array}{c}
\text { tile-union }(N, S), \\
\text { tile-union }(N, S: E), \\
\text { tile-union }(N: W, S), \\
\text { tile-union }(N: W, S: E)
\end{array}\right\}=\left\{\begin{array}{c}
N: S, \\
N: S: E, \\
N: W: S, \\
N: W: S: E
\end{array}\right\} .
\end{aligned}
$$

\subsection{Computing the Inverse of a Directional Relation}

Before we proceed, we present a useful proposition. Proposition 1 reveals the inherent symmetry in the $\mathcal{C} D R$ family and simplifies the proofs of Lemmas 1 and 2 .

Proposition 1. Consider a basic directional relation $R_{1}: \cdots: R_{k}$. Let us assume that its inverse is a directional relation that can be represented as a function of the five tiles $N, W, S, E$, and $B$, that is, inv $\left(R_{1}: \cdots: R_{k}\right)=f(N, W, S, E, B)$. Then,

1.

$$
\begin{aligned}
\operatorname{inv}\left(R_{1}^{\leftarrow}: \cdots: R_{k}^{\leftarrow}\right) & =f\left(N^{\leftarrow}, W^{\leftarrow}, S^{\leftarrow}, E^{\leftarrow}, B\right) \\
& =f(W, S, E, N, B) .
\end{aligned}
$$

2.

$$
\begin{aligned}
\operatorname{inv}\left(R_{1}^{\rightarrow}: \cdots: R_{k}^{\rightarrow}\right) & =f\left(N^{\rightarrow}, W^{\rightarrow}, S^{\rightarrow}, E^{\rightarrow}, B\right) \\
& =f(E, N, W, S, B) .
\end{aligned}
$$

3.

$$
\begin{aligned}
\operatorname{inv}\left(R_{1}^{\downarrow}: \cdots: R_{k}^{\downarrow}\right) & =f\left(N^{\downarrow}, W^{\downarrow}, S^{\downarrow}, E^{\downarrow}, B\right) \\
& =f(S, E, N, W, B) .
\end{aligned}
$$

Proof. Case 1. This is due to the symmetry of the directional relations of $\mathcal{C} D R$. To give an example, let us observe Fig. 5a. For this spatial configuration, we have $a_{1} N: W b_{1}$, $b_{1} S: E a_{1}$ and, thus, $S: E \in \operatorname{inv}(N: W)$. Now, consider rotating the configuration in Fig. 5a by $90^{\circ}$ counterclockwise (Fig. 5b). The effect of this rotation is that the tiles in our relations have also been rotated. Specifically, $N$ became $W\left(N^{\leftarrow}\right), W$ became $S\left(W^{\leftarrow}\right), S$ became $E\left(S^{\leftarrow}\right)$, and $E$ became $N\left(E^{\leftarrow}\right)$. Notice that, in Fig. 5b, we have $a_{2} W: S b_{2}, b_{2} N: E a_{2}$, and, thus, $N: E \in \operatorname{inv}(W: S)$. Note that these expressions can be derived directly from the expressions $a_{1} N: W b_{1}, b_{1} S: E a_{1}$, and $S: E \in \operatorname{inv}(N: W)$ concerning Fig. 5a by directly applying the aforementioned substitutions.

Cases 2 and 3. These cases also hold due to the symmetry of directional relations. To verify this, we have to rotate the plane clockwise by $90^{\circ}$ and $180^{\circ}$, respectively. 


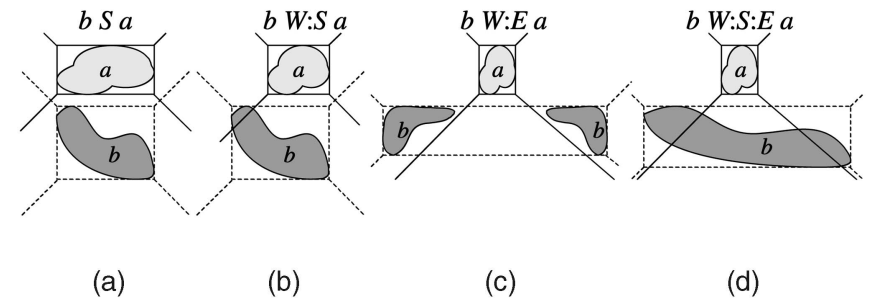

Fig. 6. Proving Lemma 1a, for $R=N$.

We now present and formally prove Lemma 1 for computing the inverse of single-tile relations.

Lemma 1. Let $R \in\{N, W, S, E\}$ be a single-tile directional relation. Then,

a. $\quad \operatorname{inv}(R)=\delta\left(R^{\leftarrow}, R^{\rightarrow}, R^{\downarrow}\right)-\left\{R^{\leftarrow}, R^{\rightarrow}\right\}$ and

b. $\quad \operatorname{inv}(B)=U_{d i r}-\{N, W, S, E\}$.

Proof. Case 1. We will first prove that the expression of Lemma 1a, holds for $R=N$, that is,

$$
\operatorname{inv}(N)=\delta(W, S, E)-\{W, E\} .
$$

Let $a$ and $b$ be two objects in $R E G^{*}$ such that $a N b$. Since $a$ is north of $b$, we can intuitively understand and easily verify that no part of $b$ can, in turn, lie north or inside the $m b b$ of object $a$. Therefore, no part of $b$ can lie inside tiles $N(a)$ and $B(a)$ and, consequently, tiles $N$ and $B$ cannot appear in directional relation $i n v(N)$, which implies that

$$
\begin{aligned}
& \operatorname{inv}(N) \subseteq \delta(W, S, E)= \\
& \{W, S, E, W: S, W: E, S: E, W: S: E\} .
\end{aligned}
$$

Let us now consider every basic relation in $\delta(W, S, E)$ and check whether it belongs to $\operatorname{inv}(N)$ or not:

1. Relation $S$. Fig. 6a demonstrates that $b S a$ is possible; thus, $S \in \operatorname{inv}(N)$.

2. Relation $W: S$. Fig. $6 \mathrm{~b}$ shows that $b W: S a$ is possible; thus, $W: S \in \operatorname{inv}(N)$.

3. Relation $S: E$. Similar with relation $W: S$, we can show that $S: E \in \operatorname{inv}(N)$.

4. Relation $W: E$. Fig. 6c depicts the not-so-obvious possibility that $b W: E a$; thus, $W: E \in \operatorname{inv}(N)$.

5. Relation $W: S: E$. Fig. 6d shows that $b W: S: E a$ is possible; thus, $W: S: E \in \operatorname{inv}(N)$.

6. Relation $W$. It is not possible to create a spatial configuration such that $b W a$; thus, $W \notin \operatorname{inv}(N)$.

7. Relation E. Similar with relation $W$, it is not possible to create a spatial configuration such that $b E a$ and, thus, $E \notin i n v(N)$.

Therefore, we have $\operatorname{inv}(N)=\delta(W, S, E)-\{W, E\}$ (M). By applying Proposition 1 to Expression (M), we also have

$$
\begin{aligned}
& i n v(W)=\delta(S, N, E)-\{S, N\}, \\
& i n v(S)=\delta(E, W, N)-\{E, W\}, \quad \text { and } \\
& i n v(E)=\delta(N, S, W)-\{N, S\} .
\end{aligned}
$$

The above expressions and Expression (M) are captured by Lemma 1a.
Case 2. In order to compute $\operatorname{inv}(B)$, we apply the same procedure as with Case 1 . Let $a$ and $b$ be two objects in $R E G^{*}$ such that $a B b$. In this case, we cannot eliminate any tiles from $\operatorname{inv}(B)$, so, as a starting point, we will consider that

$$
\operatorname{inv}(B) \subseteq U_{d i r} .
$$

By examining every basic relation in $U_{d i r}$ in the same way that we did while proving Case 1 , we conclude that $\operatorname{inv}(B)=U_{\text {dir }}-\{N, W, S, E\}$.

The following lemma can be used to compute the inverse of multitile relations:

Lemma 2. Let $R=R_{1}: \cdots: R_{k} \quad(2 \leq k \leq 5)$ be a multitile directional relation. Also, let

$$
\bar{R}=\{N, W, S, E, B\}-\left\{R_{1}, \ldots, R_{k}\right\}
$$

Then,

$$
\begin{aligned}
& \text { a. } \quad \text { inv }(R)=\delta(\bar{R}) \text { if } B \notin\left\{R_{1}, \ldots, R_{k}\right\} \text { and } \\
& \text { b. } \quad \operatorname{inv}(R)=\delta(\bar{R}, B)-\bar{R} \text { if } B \in\left\{R_{1}, \ldots, R_{k}\right\} .
\end{aligned}
$$

Proof. Case 1. We will first prove that the expression of Lemma 2a holds for $R=N: W$, that is,

$$
\operatorname{inv}(N: W)=\delta(S, E, B) .
$$

Let $a$ and $b$ be two objects in $R E G^{*}$ such that $a N: W b$. Since $a$ is north and west of $b$, we can intuitively understand and easily verify that no part of $b$ can, in turn, lie north or west of object $a$. Therefore, no part of $b$ can lie within tiles $N(a)$ and $W(a)$. Consequently, we can exclude tiles $N$ and $W$ from directional relation $\operatorname{inv}(N: W)$. Thus, as a starting point, we can use the following expression:

$$
\begin{aligned}
& \operatorname{inv}(N: W) \subseteq \delta(S, E, B)= \\
& \quad\{S, E, B, S: E, S: B, E: B, S: E: B\} .
\end{aligned}
$$

Let us now consider every basic relation in $\delta(S, E, B)$ and check whether it belongs to $\operatorname{inv}(N: W)$ or not.

1. Relation E. Fig. 7a shows that $b E a$ is possible; thus, $E \in \operatorname{inv}(N: W)$.

2. Relation $E: B$. Fig. $7 \mathrm{~b}$ illustrates that $b E: B a$ is possible; thus, $E: B \in \operatorname{inv}(N: W)$.

3. Relation $S: E$. Fig. 7c shows that $b S: E$ a is also feasible; thus, $S: E \in$ inv $(N: W)$.

4. Relation $S: E: B$. Fig. $7 d$ shows that $b S: E: B a$ is feasible; thus, $S: E: B \in \operatorname{inv}(N: W)$.

5. Relation $S$. Fig. 7e illustrates that $b S a$ is possible; thus, $S \in \operatorname{inv}(N: W)$.

6. Relation $S: B$. Fig. $7 \mathrm{f}$ shows that $b S: B$ is feasible; thus, $S: B \in \operatorname{inv}(N: W)$.

7. Relation $B$. Fig. 7g illustrates that $b B a$ is possible; thus, $B \in \operatorname{inv}(N: W)$.

Therefore, $\operatorname{inv}(N: W)=\delta(S, E, B)$ (T1). By applying Proposition 1 to Expression (T1), we have

$$
\begin{gathered}
\operatorname{inv}(W: S)=\delta(N, E, B), \operatorname{inv}(N: E)=\delta(W, S, B), \text { and } \\
\operatorname{inv}(S: E)=\delta(N, W, B) .
\end{gathered}
$$




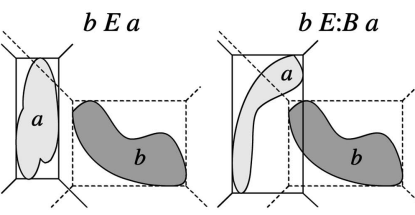

(a) (e)

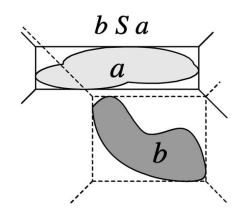

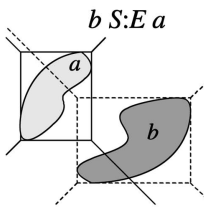

(c)

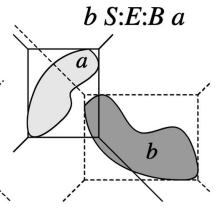

(d)

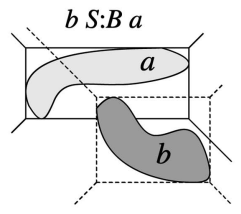

(f)

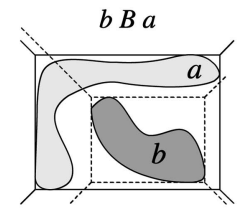

(g)
Fig. 7. Proving Lemma 2a for $R=N: W$.

The above expressions verify that Lemma $2 \mathrm{a}$ also holds for relations $W: S, N: E$, and $S: E$.

We will now prove that the expression of Lemma $2 \mathrm{a}$ holds for $R=N: S$, that is, $\operatorname{inv}(N: S)=\delta(W, E, B)$. To this end, we will follow the same procedure as with relation $N: W$.

Let $a$ and $b$ be two objects in $R E G^{*}$ such that $a N: S b$. Since no part of object $b$ can lie inside tiles $N(a)$ and $S(a)$, we can exclude tiles $N$ and $S$ from directional relation $\operatorname{inv}(N: S)$. Therefore, as a starting point, we can use expression

$$
\begin{aligned}
\operatorname{inv}(N: S) \subseteq \delta(W, E, B)= & \\
& \{W, E, B, W: E, W: B, E: B, W: E: B\} .
\end{aligned}
$$

By examining every relation in $\delta(W, E, B)$, we conclude that $\operatorname{inv}(N: S)=\delta(W, E, B)$ (T2). Furthermore, by applying Proposition 1 to Expression (T2), we can prove that Lemma 2 a also holds for relation $W: E$.

Let us now prove that the expression of Lemma $2 \mathrm{a}$ holds for $R=N: W: E$, that is, inv $(N: W: E)=\delta(S, B)$. We will follow the same procedure as with relation $N: W$.

Let $a$ and $b$ be two objects in $R E G^{*}$ such that $a N: W: E b$. Since no part of object $b$ can lie inside tiles $N(a), W(a)$, and $E(a)$, we can exclude tiles $N, W$, and $E$ from directional relation $\operatorname{inv}(N: W: E)$. Therefore, as our starting point, we can use expression

$$
\operatorname{inv}(N: W: E) \subseteq \delta(S, B)=\{S, B, S: B\} .
$$

By examining every relation in $\delta(S, B)$, we conclude that $\operatorname{inv}(N: W: E)=\delta(S, B)$ (T3). Furthermore, by applying Proposition 1 to Expression (T3), we can prove that Lemma 2a also holds for relations $N: W: S, N: S: E$, and $W: S: E$. Last, it is easy to verify that the expression of Lemma $2 \mathrm{a}$ also holds for $R=N: W: S: E$, that is, $\operatorname{inv}(N: W: S: E)=\delta(B)=B$.

In summary, we have proven that Lemma 2 a holds for all multitile relations that do not contain tile $B$.

Case 2. This case can be proven by applying the same procedure as with Case 1 . We start by verifying that the expression of Lemma 2 a holds for relations $N: B, N: W: B$,
$N: S: B, \quad N: W: E: B$, and $N: W: S: E: B$. Then, by applying Proposition 1, we can verify that Lemma 2a holds for all multitile relations that include tile $B$.

To compute the inverse of an arbitrary directional relation, we can use the following theorem in combination with Lemmas 1 and 2:

Theorem 1. Let $Q=\cup_{i=1}^{k} R_{i}$ be a directional relation in $2^{\mathcal{B}^{*}}$, where $R_{i}$ are basic directional relations. Then,

$$
\operatorname{inv}(Q)=\cup_{i=1}^{k}\left(\operatorname{inv}\left(R_{i}\right)\right) .
$$

Note that $\operatorname{inv}\left(R_{i}\right)$ can be computed using Lemmas 1 and 2.

Proof. From the definition of inverse (Definition 5), we have

$$
\operatorname{inv}(Q)=\left\{R \in \mathcal{B}^{*}:\left(\exists a, b \in R E G^{*}\right)(a Q b \wedge b R a)\right\} .
$$

Since $Q=\cup_{i=1}^{k} R_{i}$, we have $a Q b=a R_{1} b \vee \cdots \vee a R_{k} b$. Therefore,

$$
\begin{aligned}
\operatorname{inv}(Q)=\left\{R \in \mathcal{B}^{*}:\right. & \left(\exists a, b \in R E G^{*}\right) \\
& \left.\left(a R_{1} b \vee \cdots \vee a R_{k} b\right) \wedge b R a\right\} .
\end{aligned}
$$

By distributing $\wedge$ over $\vee$, we have

$$
\begin{aligned}
\operatorname{inv}(Q)=\left\{R \in \mathcal{B}^{*}:\right. & \left(\exists a, b \in R E G^{*}\right) \\
& \left.\left(a R_{1} b \wedge b R a\right) \vee \cdots \vee\left(a R_{k} b \wedge b R a\right)\right\} .
\end{aligned}
$$

Thus, $\operatorname{inv}(Q)=\cup_{i=1}^{k}\left(\operatorname{inv}\left(R_{i}\right)\right)$ holds.

\subsection{Computing the Composition of Two Directional Relations}

Before we address the composition problem, we present Proposition 2, which serves the same purpose that Proposition 1 did while studying the inverse problem, that is, it reveals the inherent symmetry in the $\mathcal{C} D R$ family and simplifies the proofs of the relevant lemmas.

Proposition 2. Consider two basic directional relations: $R_{1}=$ $R_{11}: \cdots: R_{1 k}$ and $R_{2}=R_{21}: \cdots: R_{2 m}$. Let us assume that their composition is a directional relation that can be represented as a function of the five tiles $N, W, S, E$, and $B$, that is, $R_{1} \circ R_{2}=f(N, W, S, E, B)$. Then,

1.

$$
\begin{aligned}
& R_{11}^{\leftarrow}: \cdots: R_{1 k}^{\leftarrow} \circ R_{21} q q^{\leftarrow}: \cdots: R_{2 m}^{\leftarrow}= \\
& f\left(N^{\leftarrow}, W^{\leftarrow}, S^{\leftarrow}, E^{\leftarrow}, B\right)=f(W, S, E, N, B)
\end{aligned}
$$

2.

$$
\begin{aligned}
& R_{11}^{\rightarrow}: \cdots: R_{1 k}^{\rightarrow} \circ R_{21}^{\rightarrow}: \cdots: R_{2 m}^{\rightarrow}= \\
& f\left(N^{\rightarrow}, W^{\rightarrow}, S^{\rightarrow}, E^{\rightarrow}, B\right)=f(E, N, W, S, B) .
\end{aligned}
$$

3.

$$
\begin{aligned}
& R_{11}^{\downarrow}: \cdots: R_{1 k}^{\downarrow} \circ R_{21}^{\downarrow}: \cdots: R_{2 m}^{\downarrow}= \\
& f\left(N^{\downarrow}, W^{\downarrow}, S^{\downarrow}, E^{\downarrow}, B\right)=f(S, E, N, W, B) .
\end{aligned}
$$

Proof. This is due to the symmetry of the directional relations of $\mathcal{C} D R$. 


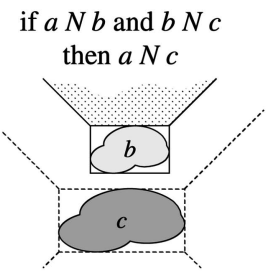

(a)

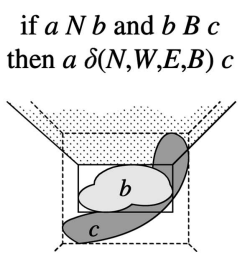

(d)

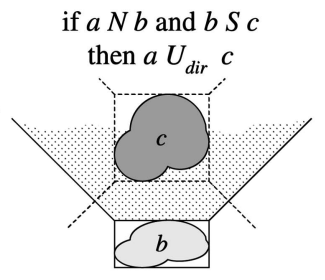

(b)

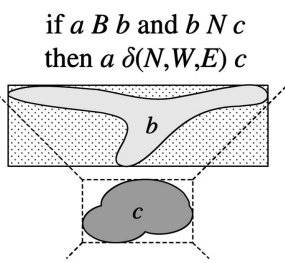

(e)

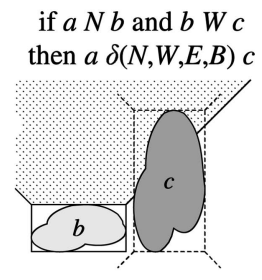

(c)

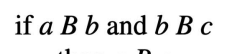
then $a B c$

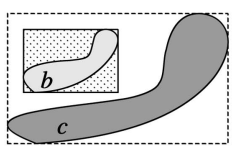

(f)
Fig. 8. Proving Lemma 3.

We will address the composition problem one step at a time. First, we consider the case of composing two singletile relations.

Lemma 3. Let $R \in\{N, W, S, E\}$ be a single-tile directional relation. Then,

$$
\begin{array}{ll}
\text { 1. } & R \circ R=R, \\
\text { 2. } & R \circ R^{\downarrow}=U_{\text {dir }} \\
\text { 3. } & R \circ R^{\leftarrow}=R \circ R^{\rightarrow}=R \circ B=\delta\left(R, R^{\leftarrow}, R^{\rightarrow}, B\right), \\
\text { 4. } & B \circ R=\delta\left(R, R^{\leftarrow}, R^{\rightarrow}\right), \text { and } \\
\text { 5. } & B \circ B=B .
\end{array}
$$

Proof. Case 1. We will first prove that the expression in Lemma 3.1 holds for $R=N$, that is, $N \circ N=N$. Let $a, b$, and $c$ be three objects in $R E G^{*}$ such that $a N b$ and $b N c$ hold. Fig. 8a presents objects $b$ and $c$ such that $b N c$. Since $a N b$, object $a$ lies inside tile $N(b)$ (the dotted area in Fig. 8a). Notice that tile $N(b)$ can only lie inside tile $N(c)$ and, consequently, object $a$ can only lie inside tile $N(c)$. More formally, we have $a \subseteq N(b) \subseteq N(c)$. In other words, if $a N b$ and $b N c$, then $a N c$ and, thus, $N \circ N=N$ (S1) holds. By applying Proposition 2 to Expression (S1), we also have

$$
W \circ W=W, E \circ E=E \text { and } S \circ S=S .
$$

All of the above expressions and Expression (S1) are captured by Lemma 3.1.

Case 2. We will first prove that the expression of Lemma 3.2 holds for $R=N$, that is, $N \circ S=U_{d i r}$. Let $a, b$, and $c$ be three objects in $R E G^{*}$ such that $a N b$ and $b S c$ hold. Fig. $8 \mathrm{~b}$ presents objects $b$ and $c$ such that $b S c$. Object $a$ lies inside tile $N(b)$ (the dotted area in Fig. 8b). Notice that area $N(b)$ can intersect with all five tiles of object $c$, namely, $N(c), W(c), S(c), E(c)$, and $B(c)$. Consequently, object $a$ can lie within any of these five tiles or any combination of them. In other words, if $a N b$ and $b S c$, then $a \delta(N, W, S, E, B) c$ or $a U_{d i r} c$ and, thus, $N \circ S=U_{\text {dir }}$ (S2) holds. By applying Proposition 2 to Expression (S2), we can easily verify that Lemma 3.2 holds.

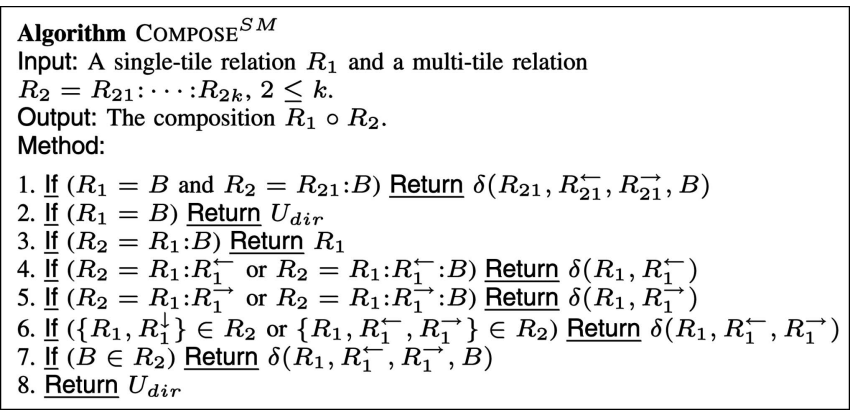

Fig. 9. Algorithm COMPOSE ${ }^{S M}$.

Case 3. We will first prove that expression $R \circ R^{\leftarrow}=$ $\delta\left(R, R^{\leftarrow}, R^{\rightarrow}, B\right)$ holds for $R \in\{N, W, S, E\}$. Fig. 8c helps us verify that the expression holds for $R=N$, that is, $N \circ W=\delta(N, W, E, B)$ (S3). Then, by applying Proposition 2 to Expression (S3), we can prove that the expression holds for every $R \in\{N, W, S, E\}$.

In a similar manner, we can also prove that $R$ o $R^{\rightarrow}=\delta\left(R, R^{\leftarrow}, R^{\rightarrow}, B\right)$ holds for $R \in\{N, W, S, E\}$. We will now prove that expression $R \circ B=\delta\left(R, R^{\leftarrow}, R^{\rightarrow}, B\right)$ holds for $R \in\{N, W, S, E\}$. Fig. 8d helps us verify that the expression holds for $R=N$, that is, $N \circ B=$ $\delta(N, W, E, B)(\mathrm{S} 4)$. Then, by applying Proposition 2 to Expression (S4), we can prove that the expression holds for every $R \in\{N, W, S, E\}$.

Therefore, we have proven that Lemma 3.3 holds.

Case 4. Fig. 8e helps us verify that expression $B \circ R=$ $\delta\left(R, R^{\leftarrow}, R^{\rightarrow}\right)$ holds for $R=N$, that is, $B \circ N=\delta(N, W, E)$ (S5). By applying Proposition 2 to Expression (S5), we can verify that Lemma 3.4 holds.

Case 5. This case is trivial and Fig. 8f helps us verify that $B \circ B=B$.

We will now turn our attention to the composition of a single-tile directional relation with a multitile directional relation. To this end, we use the COMPOSE ${ }^{S M}$ Algorithm (Fig. 9). The algorithm takes as inputs a single-tile directional relation $R_{1}$ and a multitile relation $R_{2}=R_{21}$ : $\cdots: R_{2 k}(k \geq 2)$ and returns the composition $R_{1} \circ R_{2}$. The following is an example of the COMPOSE ${ }^{S M}$ Algorithm in operation:

Example 5. Let $R_{1}=N$ and $R_{2}=N: B=R_{1}: B$. Using the COMPOSE $^{S M}$ Algorithm (Line 3), we have $N \circ N: B=N$. This can be verified using Fig. 10c.

The following lemma establishes the correctness of the COMPOSE $^{S M}$ Algorithm:

Lemma 4. Let $R_{1}$ be a single-tile directional relation and $R_{2}=$ $R_{21}: \cdots: R_{2 k}$ be a multitile directional relation. Then, $R_{1} \circ R_{2}$ can be computed by the Algorithm COMPOSE ${ }^{S M}$.

Proof. Every line of the algorithm computes the composition of a set of pairs of basic directional relations. Particularly, lines 1 and 2 compute the composition for $R_{1}=B$. The rest of the algorithm computes the composition for $R_{1} \in\{N, W, S, E\}$. Therefore, we will examine each line of the algorithm individually and verify that it correctly computes the relevant composition. 


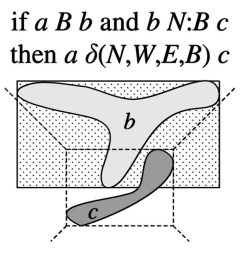

(a)

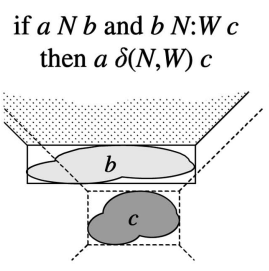

(d)

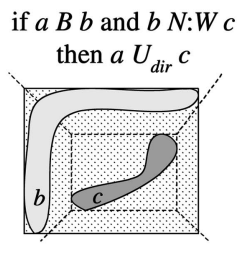

(b)

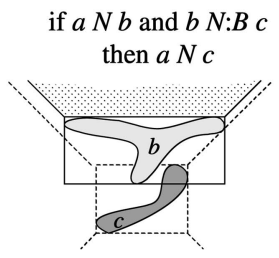

(c)

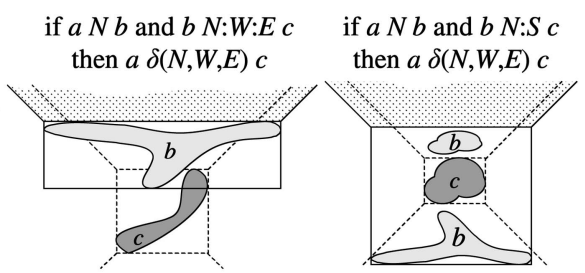

(e)

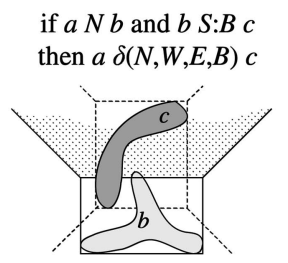

(g)

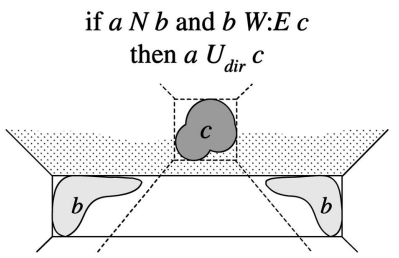

(h)

Fig. 10. Proving Lemma 4.

Line 1. This If statement states that

$$
B \circ R_{21}: B=\delta\left(R_{21}, R_{21}^{\leftarrow}, R_{21}^{\rightarrow}, B\right),
$$

where $R_{21} \in\{N, W, S, E\}$. We will first prove that this expression holds for $R_{21}=N$, that is,

$$
B \circ N: B=\delta(N, W, E, B) .
$$

Let $a, b$, and $c$ be three objects in $R E G^{*}$ such that $a B b$ and $b N: B c$ hold. Fig. 10a presents objects $b$ and $c$ such that $b N: B c$. Since $a B b$, object $a$ lies inside tile $B(b)$ (the dotted area in Fig. 10a). Notice that area $N(b)$ can intersect with tiles $N(c), W(c), E(c)$, and $B(c)$. Consequently, object $a$ can lie within any of these four tiles or any combination of them. In other words, if $a B b$ and $b N: B c$, then $a \delta(N, W, E, B) c$. Thus, $N \circ N: B=$ $\delta(N, W, E, B)$ (E1) holds. By applying Proposition 2 to Expression (E1), we also have

$$
\begin{gathered}
B \circ W: B=\delta(N, W, S, B), B \circ E: B=\delta(N, S, E, B), \text { and } \\
B \circ S: B=\delta(W, S, E, B) .
\end{gathered}
$$

All of the above expressions and Expression (E1) are captured by Line 1 of the Algorithm COMPOSE ${ }^{S M}$.

Line 2. The condition of this If statement is satisfied when $R_{1}=B$, and $R_{2} \neq\{N: B, \bar{W}: B, S: B, E: B\}$; otherwise, the condition of line 1 would have been satisfied. Line 2 holds for $R_{2}=N: W$, that is, $B \circ N: W=U_{d i r}$. This can be verified using Fig. 10b. Also by using Fig. 10b, we can verify that the composition of $B$ with any relation that is made up of at least two adjacent tiles (that is, $\left.R_{2} \neq\{N: B, W: B, S: B, E: B\}\right)$ is equal to $U_{d i r}$.

Line 3. This If statement states that $R_{1} \circ R_{1}: B=R_{1}$, $R_{1} \in\{N, W, S, E\}$. To prove that this expression holds for $R_{1}=N$, that is, $N \circ N: B=N$ (E2), we use Fig. 10c. Then, by applying Proposition 2 to Expression (E2), we can verify that Line 3 is correct for all $R_{1} \in\{N, W, S, E\}$.

Line 4. The condition of this If statement is satisfied when $R_{2} \in\left\{R_{1}: R_{1}^{\leftarrow}, R_{1}: R_{1}^{\leftarrow}: B\right\}$. We will only prove that Line 4 is correct for $R_{2}=R_{1}: R_{1}^{\leftarrow}$, that is, $R_{1} \circ R_{1}: R_{1}^{\leftarrow}=\delta\left(R_{1}: R_{1}^{\leftarrow}\right)$. The proof for $R_{2}=R_{1}: R_{1}^{\leftarrow}: B$ is similar. Line 4 holds for $R_{1}=N$, that is, $N \circ N: W=$ $\delta(N, W)$ (E3). This can be verified using Fig. 10d. By applying Proposition 2 to Expression (E3), we can also verify that Line 4 is correct for all $R_{1} \in\{N, W, S, E\}$.

Line 5. The proof is similar to the proof of Line 4 .

Line 6. The condition of this If statement is satisfied when relation $R_{2}$ contains tiles $\left\{\overline{R_{1}}, R_{1}^{\downarrow}\right\}$ or $\left\{R_{1}, R_{1}^{\leftarrow}, R_{1}^{\rightarrow}\right\}$. We will first prove that line 6 holds for $R_{1}=N$. Then, $R_{2}$ contains tiles $\{N, S\}$ or $\{N, W, E\}$. We will concentrate on the two most representative relations of this group, namely, $R_{2}=N: S$ and $R_{2}=N: W: E$, since the proofs for the other relations of the group are almost identical to one of these two. Fig. 10e helps us verify that $N \circ N: W$ : $E=\delta(N, W, E) \quad(\mathrm{E} 4)$ and Fig. 10f that $N \circ N: S=$ $\delta(N, W, E)$ (E5). Then, by applying Proposition 2 to Expressions (E4) and (E5), we can verify that line 6 holds for all $R_{1} \in\{N, W, S, E\}$.

Line 7. The If condition of this statement requires that $B \in\left\{R_{21}, \ldots, \bar{R}_{2 k}\right\}$. However, for the algorithm to reach line 7 , the conditions of the If statements in lines 3-6 must have not been satisfied. These four statements provide the composition for all relations $R_{2}$ that contain tile $R_{1}$. Therefore, relation $R_{2}$ includes tile $B$ but not tile $R_{1}$. In other words,

$$
\begin{aligned}
R_{2} \in\{ & R_{1}^{\leftarrow}: B, R_{1}^{\rightarrow}: B, R_{1}^{\downarrow}: B, R_{1}^{\leftarrow}: R_{1}^{\rightarrow}: B, \\
& \left.R_{1}^{\leftarrow}: R_{1}^{\downarrow}: B, R_{1}^{\rightarrow}: R_{1}^{\downarrow}: B, R_{1}^{\leftarrow}: R_{1}^{\rightarrow}: R_{1}^{\downarrow}: B\right\} .
\end{aligned}
$$

We will concentrate on relations of the form $R_{2}=R_{1}^{\downarrow}: B$ and prove that $R_{1} \circ R^{\downarrow}: B=\delta\left(R_{1}, R_{1}^{\leftarrow}, R_{1}^{\rightarrow}, B\right)$ (E6) holds (the proofs for the rest of the relations are similar). Expression (E6) holds for $R_{1}=N$, that is,

$$
N \circ S: B=\delta(N, W, E, B) .
$$

This can be verified using Fig. 10g. Then, by applying Proposition 2, we can prove that Expression (E6) holds for every $R_{1} \in\{N, W, S, E\}$.

Line 8. If the execution of the algorithm reaches line 8, then relation $R_{2}$ cannot contain tiles $R_{1}$ and $B$, that is, $R_{2} \in\left\{R_{1}^{\leftarrow}: R_{1}^{\rightarrow}, R_{1}^{\leftarrow}: R_{1}^{\downarrow}, R_{1}^{\rightarrow}: R_{1}^{\downarrow}, R_{1}^{\leftarrow}: R_{1}^{\rightarrow}: R_{1}^{\downarrow}\right\}$. When tile $R_{1}^{\downarrow}$ is present in relation $R_{2}$, the proof is similar to the proof of Lemma 3.2, which states that $R_{1} \circ R_{1}^{\downarrow}=U_{\text {dir }}$, so we will not consider these relations. Instead, we will prove expression $R_{1} \circ R_{1}^{\leftarrow}: R_{1}^{\rightarrow}=U_{d i r}$, which is not apparent. Fig. 10h shows that the expression holds for $R_{1}=N$, that is, $N \circ W: E=U_{d i r}$. Then, by applying Proposition 2, we can prove that line 8 is correct for all $R_{1} \in\{N, W, S, E\} . \square$

Summarizing our progress so far, we have presented Lemma 3, which can be used to compute the composition of two single-tile relations, and then the Algorithm 


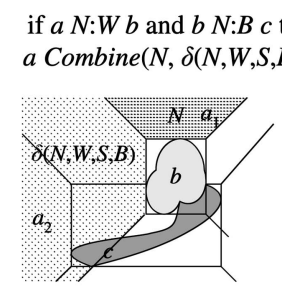

(a) if $a W: B b$ and $b N c$ then a Combine $(\delta(N, W, S, B), \delta(N, E)) c$ or a Combine $(\delta(N, W), \delta(N, W, E)) c$

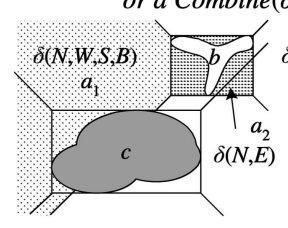

(b)

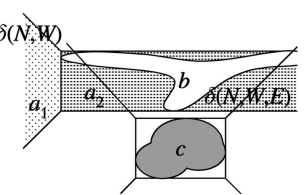

(c)
Fig. 11. Illustrations of Examples 6 and 7.

COMPOSE ${ }^{S M}$, which provides the composition of a singletile relation and a multitile relation. In other words, we are able to compute the composition of a single-tile directional relation and a basic (single-tile or multitile) directional relation. The next logical step is to address the problem of composing a multitile directional relation and a basic relation. Let us study two specific examples that will help us understand the method used to compute the composition of such relations.

Example 6. Let us compute $N: W \circ N: B$. Let $a, b$, and $c$ be three objects in $R E G^{*}$ such that $a N: W b$ and $b N: B$ c. To compute $N: W \circ N: B$, we have to find all possible relations between $a$ and $c$. According to Definition 4, $a N$ : $W b$ implies that there exist objects $a_{1}$ and $a_{2}$ such that $a_{1} N b, a_{2} W b$, and $a=a_{1} \cup a_{2}$. We can handle the composition problem for each of the two components of object $a$ separately and then use the corresponding results to create the directional relation $N: W \circ N: B$.

Fig. 11a shows two objects $b$ and $c$ such that $b N: B c$. The heavily dotted area corresponds to tile $N(b)$, where object $a_{1}$ lies at $\left(a_{1} N b\right)$, and the lightly dotted area corresponds to tile $W(b)$, where object $a_{2}$ lies at $\left(a_{2} W b\right)$. Since $a_{1} N b$ and $b N: B c$, we have $a_{1} N \circ N: B c$ and, using the Algorithm COMPOSE ${ }^{S M}$, we can compute that $a_{1} N c$ (see also Fig. 11a). Similarly, since $a_{2} W b$ and $b N: B c$, we have $a_{2} \delta(N, W, S, B) c$.

Let us see how we can use these results to calculate the possible relations between $a$ and $c$. Since $a=a_{1} \cup a_{2}$, if, for example, $a_{1} N c$ and $a_{2} W: S c$, then we have a tile-union $(N, W: S) c$ or a $N: W: S c$. Likewise, if $a_{1} N c$ and $a_{2} N: W: B c$, then a tile-union $(N, N: W: B) c$ or a $N: W: B c$. Therefore, directional relation $N: W \circ N: B$ includes all basic relations created by taking the tile-union of each relation in $\{N\}$ with every relation in $\delta(N, W, S, B)$. In other words,

$$
N: W \circ N: B=\operatorname{Combine}(N, \delta(N, W, S, B)) .
$$

The result, as well as the procedure that we have used in order to compute $N: W \circ N: B$, can be captured by expression $N: W \circ N: B=$ Combine $(N \circ N: B, W \circ N: B)$. One could be tempted to generalize this expression and use it to compute the composition of any two relations:

$$
R_{11} \because \cdots: R_{1 k} \circ R_{2}=\text { Combine }\left(R_{11} \circ R_{2}, \ldots, R_{1 k} \circ R_{2}\right),
$$

but, unfortunately, it does not always produce the correct result. Let us see another example that will help clarify why the aforementioned expression fails.
Example 7. Let us compute $W: B \circ N$. If we use Expression (C), then we have $W: B \circ N=$ Combine $(W \circ N, B \circ N)$. Since $W: S \in C$ Combine $(W \circ B, W \circ N)$, it follows from the above equation that there exists a spatial configuration such that $a W: B b, b N c$, and $a W: S c$. It is easy to verify that such a configuration does not exist; thus, Expression (C) cannot be applied to $W: B \circ N$.

To compute the correct composition, let $a, b$, and $c$ be three objects in $R E G^{*}$ such that $a W: B b$ and $b N c$. According to Definition $4, a W: B b$ implies that there exist objects $a_{1}$ and $a_{2}$ such that $a_{1} W b, a_{2} B b$, and $a=a_{1} \cup a_{2}$. Figs. $11 \mathrm{~b}$ and $11 \mathrm{c}$ depict two spatial configurations involving objects $b$ and $c$ such that $b N c$. In both cases, the lightly dotted area corresponds to tile $W(b)$ (that is, the area where object $a_{1}$ lies), whereas the heavily dotted area corresponds to tile $B(b)$ (that is, the area where object $a_{2}$ lies):

- For the configuration of Fig. $11 \mathrm{~b}$, we have $a_{1} \delta(N, W, S, B) c$ and $a_{2} \delta(N, E) c$. Thus, we have a Combine $(\delta(N, W, S, B), \delta(N, E)) c$ and

$$
\text { Combine }(\delta(N, W, S, B), \delta(N, E)) \subseteq W: B \circ N .
$$

- For the configuration of Fig. 11c, we have $a_{1} \delta(N, W) c$ and $a_{2} \delta(N, W, E) c$. Thus, we have a Combine $(\delta(N, W), \delta(N, W, E)) c$ and

$$
\text { Combine }(\delta(N, W), \delta(N, W, E)) \subseteq W: B \circ N .
$$

In summary, we have

$$
\begin{aligned}
& \text { Combine }(\delta(N, W, S, B), \\
& \delta(N, E)) \cup \text { Combine }(\delta(N, W), \\
& \delta(N, W, E)) \subseteq W: B \circ N .
\end{aligned}
$$

It is not hard to verify that any other spatial configuration such that $a W: B b$ and $b N c$ would produce composition results that are a subset of those produced by the configurations of Figs. $11 \mathrm{~b}$ and 11c. Thus,

$$
\begin{aligned}
& W: B \circ N=\text { Combine }(\delta(N, W, S, B), \\
& \delta(N, E)) \cup \text { Combine }(\delta(N, W), \delta(N, W, E)),
\end{aligned}
$$

or, equivalently,

$$
\begin{aligned}
W: B \circ N & =\text { Combine }(W \circ N, B \circ N) \\
& -\{W: S, W: B, W: S: B\} .
\end{aligned}
$$

Summarizing Examples 6 and 7, we can distinguish two cases. For some pairs of relations $R_{1}=R_{11}: \cdots: R_{1 k}$ and $R_{2}$, like $N: W$ and $N: B$ of Example 6, Expression (C) can be applied directly. For the other cases, there are pairs, like $W: B$ and $N$ of Example 7, to which Expression (C) cannot be applied directly. Fortunately, as we will see later, we always have

$$
R_{1} \circ R_{2}: \subseteq \text { Combine }\left(R_{11} \circ R_{2}, \ldots, R_{1 k} \circ R_{2}\right) .
$$

Based on this observation, we present the Algorithm COMPOSE $^{M}$ (Fig. 12), which can be used to compute the composition of a multitile directional relation and a basic directional relation. The Algorithm $\operatorname{COMPOSE}^{M}$ takes as inputs a multitile directional relation $R_{1}=R_{11}: \cdots$ : $R_{1 k}(k \geq 2)$ and a basic relation $R_{2}$. Initially, the algorithm 


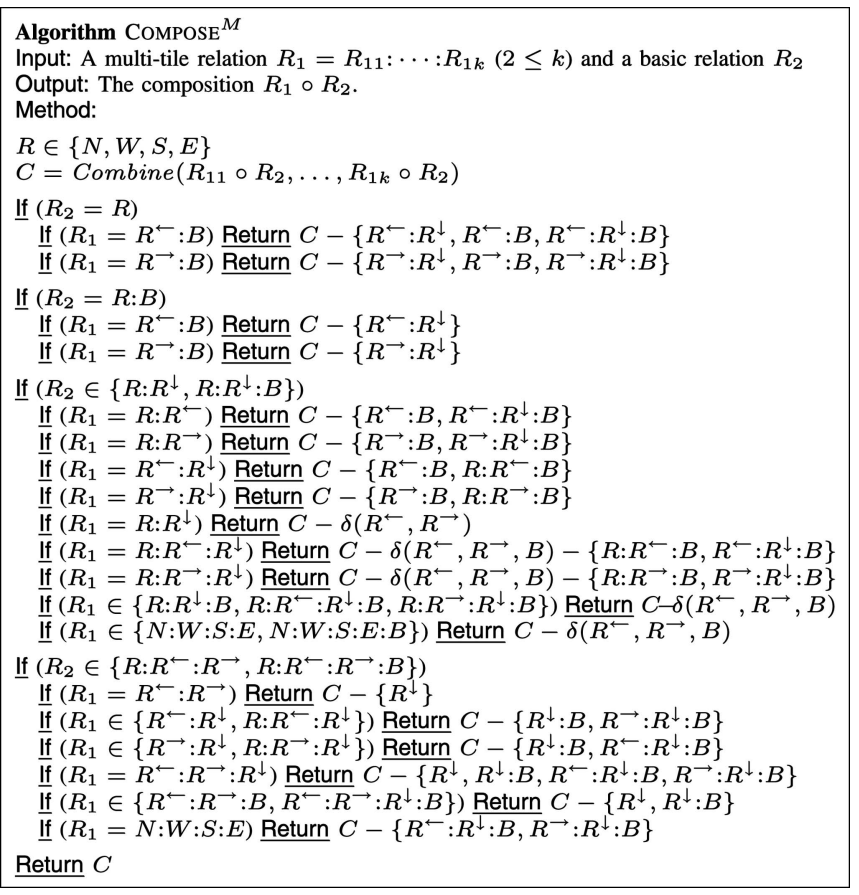

Fig. 12. Algorithm COMPOSE ${ }^{M}$.

computes set $C=$ Combine $\left(R_{11} \circ R_{2}, \ldots, R_{1 k} \circ R_{2}\right)$. Then, it removes from set $C$ all relations that cannot belong to the composition $R_{1} \circ R_{2}$.

The following lemma demonstrates the correctness of the Algorithm COMPOSE ${ }^{M}$.
Lemma 5. Let $R_{1}$ and $R_{2}$ be two basic directional relations. Then, $R_{1} \circ R_{2}$ can be computed by Algorithm COMPOSE ${ }^{M}$.

Proof. To demonstrate the correctness of the Algorithm COMPOSE $^{M}$, we will present the steps that we followed in order to create it. As we discussed earlier, for some relation pairs $R_{1}=R_{11}: \cdots: R_{1 k}$ and $R_{2}$, we can directly compute their composition by using Expression (C) (Example 6), whereas, for other pairs, we must compute their composition from first principals (like in Example 7). For these pairs, the composition is equal to a subset of Combine $\left(R_{11} \circ R_{2}, \ldots, R_{1 k} \circ R_{2}\right)$ and therefore can be described using an expression of the form $R_{1} \circ R_{2}=$ Combine $\left(R_{11} \circ R_{2}, \ldots, R_{1 k} \circ R_{2}\right)-S$, where $S$ is a set of basic directional relations.

Based on this observation, we present Table 1 . This table presents the composition of all 26 multitile relations and relations $B, N, N: B, N: W, N: W: B, N: W: E, N: W: E: B$, $N: W: S: E$, and $N: W: S: E: B$. In Table 1 , we use a star $(\star)$ to denote that the composition can be computed using expression $R_{1} \circ R_{2}=$ Combine $\left(R_{11} \circ R_{2}, \ldots, R_{1 k} \circ R_{2}\right)$. In cases where the composition is computed using expression $R_{1} \circ R_{2}=$ Combine $\left(R_{11} \circ R_{2}, \ldots, R_{1 k}\right)-S$, we simply write the set $S$. The complete transitivity table can be derived from Table 1 by using Proposition 2 .

The structure of the Algorithm COMPOSE ${ }^{M}$ reflects the results of Table 1 . The composition of most relation pairs is equal to $C$, whereas a handful of pairs produce results equal to $C-S$. Thus, the algorithm is mainly a list of rules describing these exceptions. Let us now see how these rules can be derived from Table 1 . Consider,

TABLE 1

Proving Lemma 5

\begin{tabular}{|c|c|c|c|c|c|c|c|c|}
\hline$R_{1} / R_{2}$ & $B$ & $N$ & $N: B$ & $\begin{array}{c}N: W \\
N: W: B\end{array}$ & $N: S$ & $N: S: B$ & $\begin{array}{c}N: W: E \\
N: W: E: B\end{array}$ & $\begin{array}{c}N: W: S: E, \\
N: W: S: E: B\end{array}$ \\
\hline$N: W$ & $\star$ & $\star$ & $\star$ & $\star \star$ & $\{W: B, W: S: B\}$ & $\{W: B, W: S: B\}$ & $\star$ & $\star \star$ \\
\hline$N: S$ & $\star$ & $\star$ & $\star$ & $\star$ & $\{W, E, W: E\}$ & $\{W, E, W: E\}$ & $\star$ & $\star$ \\
\hline$N: E$ & $\star$ & $\star$ & $\star$ & $\star$ & $\{E: B, S: E: B\}$ & $\{E: B, S: E: B\}$ & $\star$ & $\star$ \\
\hline$N: B$ & $\star$ & $\star$ & $\star$ & $\star$ & $\star$ & $\star$ & $\star$ & $\star$ \\
\hline$W: S$ & $\star$ & $\star$ & $\star$ & $\star$ & $\{W: B, N: W: B\}$ & $\{W: B, N: W: B\}$ & $\{S: B, S: E: B\}$ & $\star$ \\
\hline$W: E$ & $\star$ & $\star$ & $\star$ & $\star$ & $\star$ & $\star$ & $\{S\}$ & $\star$ \\
\hline$W: B$ & 夫 & $\begin{array}{c}\{W: S, W: B \\
W: S: B\}\end{array}$ & $\{W: S\}$ & 夫 & $\star$ & * & * & $\star$ \\
\hline$S: E$ & $\star$ & $\star$ & $\star$ & $\star$ & $\{E: B, N: E: B\}$ & $\{E: B, N: E: B\}$ & $\{S: B, W: S: B\}$ & $\star$ \\
\hline$S: B$ & $\star$ & $\star$ & $\star$ & $\star$ & $\star$ & $\star$ & $\star$ & $\star$ \\
\hline$E: B$ & $\star$ & $\begin{array}{c}\{S: E, E: B \\
S: E: B\}\end{array}$ & $\{S: E\}$ & $\star$ & $\star$ & $\star$ & $\star$ & * \\
\hline$N: W: S$ & * & 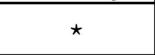 & * & * & $\begin{array}{c}\{W, E, W: E, W: B, E: B \\
N: W: B, W: S: B, W: E: B\}\end{array}$ & $\begin{array}{c}\{W, W: E, W: B, E: B \\
N: W: B, W: S: B, W: E: B\}\end{array}$ & $\{S: E: B\}$ & $\star$ \\
\hline$N: W: E$ & $\star$ & $\star$ & $\star$ & $\star$ & $\star$ & $\star \quad$ & $\star$ & $\star$ \\
\hline$N: W: B$ & $\star$ & $\star$ & $\star$ & $\star$ & $\star$ & $\star$ & $\star$ & $\star$ \\
\hline$N: S: E$ & * & $\star$ & * & * & $\begin{array}{l}\{W, E, W: E, W: B, E: B, \\
N: E: B, S: E: B, W: E: B\}\end{array}$ & $\begin{array}{c}\{E, W: E, W: B, E: B, \\
N: E: B, S: E: B, W: E: B\}\end{array}$ & $\{W: S: B\}$ & * \\
\hline$N: S: B$ & $\star$ & $\star$ & $\star$ & $\star$ & $\begin{array}{c}\{W, E, W: E, W: B \\
E: B, W: E: B\}\end{array}$ & $\begin{array}{c}\{W, E, W: E, W: B \\
E: B, W: E: B\}\end{array}$ & $\star$ & $\star$ \\
\hline$N: E: B$ & $\star$ & $\star$ & $\star$ & $\star$ & $\star$ & $\star$ & $\star$ & $\star$ \\
\hline$W: S: E$ & 夫 & $\star$ & * & * & 夫 & * & $\begin{array}{c}\{S, S: B, W: S: B \\
S: E: B\}\end{array}$ & * \\
\hline$W: S: B$ & $\star$ & $\star$ & $\star$ & $\star$ & $\star$ & $\star$ & $\star$ & $\star \star$ \\
\hline$W: E: B$ & $\star$ & $\star$ & $\star$ & $\star$ & $\star$ & $\star$ & $\{S, S: B\}$ & $\star$ \\
\hline$S: E: B$ & $\star$ & $\star$ & $\star$ & $\star$ & $\star$ & $\star$ & $\star$ & $\star$ \\
\hline$N: W: S: E$ & 夫 & $\star$ & * & * & $\begin{array}{c}\{W, E, W: E, W: B, \\
E: B, W: E: B\}\end{array}$ & $\begin{array}{c}\{W: E, W: B \\
E: B, W: E: B\}\end{array}$ & $\{W: S: B, S: E: B\}$ & * \\
\hline$N: W: S: B$ & 夫 & $\star$ & $\star$ & $\star$ & $\begin{array}{c}\{W, E, W: E, W: B \\
E: B, W: E: B\}\end{array}$ & $\begin{array}{c}\{W, W: E, W: B \\
E: B, W: E: B\}\end{array}$ & $\star$ & $\star$ \\
\hline$N: W: E: B$ & $\star$ & $\star$ & $\star$ & $\star$ & $\star$ & $\star$ & $\star$ & $\star$ \\
\hline$N: S: E: B$ & 夫 & $\star$ & $\star$ & $\star$ & $\begin{array}{c}\{W, E, W: E, W: B \\
E: B, W: E: B\}\end{array}$ & $\begin{array}{c}\{E, W: E, W: B \\
E: B, W: E: B\}\end{array}$ & $\star$ & $\star$ \\
\hline$W: S: E: B$ & $\star$ & $\star$ & $\star$ & $\star$ & $\star$ & $\star$ & $\{S, S: B\}$ & $\star$ \\
\hline$N: W: S: E: B$ & $\star$ & $\star$ & $\star$ & * & $\begin{array}{c}\{W, E, W: E, W: B \\
E: B, W: E: B\}\end{array}$ & $\begin{array}{c}\{W: E, W: B, E: B, \\
W: E: B\}\end{array}$ & $\star$ & * \\
\hline
\end{tabular}


for instance, the composition of relations $W: B$ and $N$. According to Table 1, we have

$$
\begin{aligned}
W: B \circ N & =\text { Combine }(W \circ N, B \circ N) \\
& -\{W: S, W: B, W: S: B\} .
\end{aligned}
$$

By applying Proposition 2 to this expression, we conclude that

$$
\begin{aligned}
S: B \circ W & =\text { Combine }(S \circ W, B \circ W) \\
& -\{S: E, S: B, S: E: B\}, \\
N: B \circ E= & C \text { Combine }(N \circ E, B \circ E) \\
& -\{N: W, N: B, N: W: B\}, \\
E: B \circ S= & C o m b i n e(E \circ S, B \circ S) \\
& -\{N: E, E: B, N: E: B\} .
\end{aligned}
$$

We can easily verify that the above expressions are equivalent to this single expression:

$$
\begin{aligned}
& R^{\leftarrow}: B \circ R=\text { Combine }\left(R^{\leftarrow} \circ R, B \circ R\right)- \\
& \quad\left\{R^{\leftarrow}: R^{\downarrow}, R^{\leftarrow}: B, R^{\leftarrow}: R^{\downarrow}: B\right\}, \quad R \in\{N, W, S, E\} .
\end{aligned}
$$

The final version of the algorithm, as presented in Fig. 12, contains all of the rules that can be derived from Table 1 as If statements.

The following example demonstrates how the Algorithm COMPOSE $^{M}$ is used.

Example 8. The four outer If statements of the Algorithm COMPOSE $^{M}$ regard the pattern of relation $R_{2} . R_{2}=R$ implies that $R_{2}$ consists of a single peripheral tile. $R_{2}=$ $R: B$ implies that $R_{2}$ consists of a single peripheral tile and tile $B$. Similarly, $R_{2} \in\left\{R: R^{\downarrow}, R: R^{\downarrow}: B\right\}$ implies that $R_{2}$ consists of two nonadjacent tiles and, possibly, tile $B$, whereas $R_{2} \in\left\{R: R^{\leftarrow}: R^{\rightarrow}, R: R^{\leftarrow}: R^{\rightarrow}: B\right\}$ implies that $R_{2}$ consists of three adjacent peripheral tiles and, possibly, tile $B$. For instance, the pattern of relation $R_{2}=N: S$ is $R: R^{\downarrow}$, where $R=N$. Having determined the pattern of relation $R_{2}$ and assigned a value to $R$, we proceed, if necessary, to the inner If statements and substitute the value of $R$ that we determined. For example, let us consider the composition $N: W \circ N: S$. As we mentioned earlier, the pattern of relation $R_{2}$ is $R: R^{\downarrow}$, where $R=N$. As a consequence, the condition of the third outer If statement is satisfied, so we proceed to the relevant inner If statements. By substituting $R \rightarrow N$, we notice that the first inner If statement is satisfied, since $R_{1}=R: R^{\leftarrow}=N: W$. Therefore, we have

$$
\begin{aligned}
N: W \circ N: S= & C o m b i n e(N \circ N: S, W \circ N: S) \\
& -\{W: B, W: S: B\} .
\end{aligned}
$$

To compute the composition of two arbitrary directional relations, we use the following theorem:

Theorem 2. Let $Q_{1}=\cup_{i=1}^{k} R_{1 i}$ and $Q_{2}=\cup_{j=1}^{m} R_{2 j}$ be two directional relations in $2^{\mathcal{B}^{*}}$, where all $R_{1 i}, R_{2 j}$ are basic directional relations. Then,

$$
R_{1} \circ R_{2}=\left\{R \in \mathcal{B}^{*}: R \in R_{1 i} \circ R_{2 j}\right\} .
$$

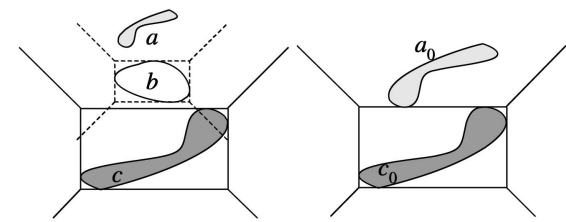

(a)

(b)

Fig. 13. Illustrations of Example 9.

Note that $R_{1 i} \circ R_{2 j}$ can be computed using Lemmas 3, 4, and 5 .

Proof. Based on Definition 7, we have

$$
\begin{aligned}
Q_{1} \circ Q_{2}=\left\{R \in \mathcal{B}^{*}:\right. & \left(\exists a, b, c \in R E G^{*}\right) \\
& \left.\left(a Q_{1} b \wedge b Q_{2} c \wedge a R c\right)\right\} .
\end{aligned}
$$

Since $Q_{1}=\cup_{i=1}^{k} R_{1 i}$ and $Q_{2}=\cup_{i=1}^{m} R_{2 j}$, we have

$$
\begin{aligned}
& a Q_{1} b=a R_{11} b \vee \cdots \vee a R_{1 k} b \text { and } \\
& b Q_{2} c=b R_{21} c \vee \cdots \vee b R_{2 m} c .
\end{aligned}
$$

Therefore,

$$
\begin{array}{r}
Q_{1} \circ Q_{2}=\left\{R \in \mathcal{B}^{*}:\left(\exists a, b, c \in R E G^{*}\right)\left(a R_{11} b \vee \cdots\right.\right. \\
\left.\left.\vee a R_{1 k} b\right) \wedge\left(b R_{21} c \vee \cdots \vee b R_{2 m} c\right) \wedge a R c\right\} .
\end{array}
$$

Finally, by distributing $\wedge$ and $\vee$, we have

$$
\begin{aligned}
Q_{1} \circ Q_{2}= & \left\{R \in \mathcal{B}^{*}:\left(\exists a, b, c \in R E G^{*}\right)\right. \\
& \left(\bigvee_{i, j}\left(a R_{1 i} b \wedge b R_{2 j} c \wedge a R c\right)\right\} \\
= & \left\{R \in \mathcal{B}^{*}: R \in R_{1 i} \circ R_{2 j}\right\} .
\end{aligned}
$$

Let us now leave the consistency-based definition of composition and consider the standard notion of existential composition from the set theory (Definition 6). Similarly to many models of spatial relations [8], [33], [17], the language of $\mathcal{C} D R$ is not expressive enough to capture the binary relation, which is the result of the existential composition of directional relations. This is illustrated by the following example:

Example 9. Consider object variables $a, b$, and $c$ and directional relations $a N b$ and $b N c$. The only directional relation implied by these two constraints is $a N c$ (see Fig. 13a). This is captured by the fact that $N \circ N=N$ (see Lemma 3). Let us now assume that $(N ; N)=N$ also holds. Then, for each pair of objects $a_{0}$ and $c_{0}$ such that $a_{0} N c_{0}$, there exists an object $b_{0} \in R E G^{*}$ such that $a_{0} N b_{0}$ and $b_{0} N c_{0}$. However, Fig. 13b shows two such objects, $a_{0}$ and $c_{0}$, such that $a_{0} N c_{0}$ and it is impossible to find an object $b_{0} \in R E G^{*}$ such that $a_{0} N b_{0}$ and $b_{0} N c_{0}$.

If we consider Fig. 13b, we will notice that the semantics of existential composition imply that object $a$ lies completely on the north tile of $c$ (that is, $a N c$ holds), and the mbbs of objects $a$ and $c$ do not touch. Intuitively, the second constraint is not expressible in the language of directional relations presented in Section 2. It is an open question to define an appropriate set of relations 
that could be used to augment the language of $\mathcal{C} D R$ such that the constraints needed to define the result of existential composition are expressible.

\section{Conclusions}

In this paper, we have introduced a family of directional relation models. We have formally defined the relations that can be expressed in the family and studied the inverse and the composition (consistency-based and existential) of directional relations. We have presented methods to compute the inverse and consistency-based composition, while we have demonstrated that the result of existential composition cannot be expressed. The aforementioned methods apply to all of the models of the family. Further research could concentrate on the extension of the $\mathcal{C} D R$ language so that existential composition is definable, as well as the study of algorithms for 1) computing the minimal network of a set of directional constraints, 2) for enforcing consistency, and 3) performing variable elimination (a task that relates to existential composition).

\section{REFERENCES}

[1] M. Egenhofer, "Reasoning about Binary Topological Relationships," Proc. Second Int'l Symp. Large Spatial Databases (SSD '91), pp. 143-160, 1991.

[2] A. Frank, "Qualitative Spatial Reasoning about Distances and Directions in Geographic Space," J. Visual Languages and Computing, vol. 3, pp. 343-371, 1992.

[3] Z. Cui, A. Cohn, and D. Randell, "Qualitative and Topological Relationships in Spatial Databases," Proc. Third Int'l Symp. Large Spatial Databases (SSD '93), 1993.

[4] J. Renz and B. Nebel, "On the Complexity of Qualitative Spatial Reasoning: A Maximal Tractable Fragment of the Region Connection Calculus," Artificial Intelligence, vols. 1-2, pp. 95-149, 1999.

[5] P. Rigaux, M. Scholl, and A. Voisard, Spatial Data Bases. Morgan Kaufmann, 2001.

[6] D. Papadias, Y. Theodoridis, T. Sellis, and M. Egenhofer, "Topological Relations in the World of Minimum Bounding Rectangles: A Study with R-Trees," Proc. ACM SIGMOD Int'l Conf. Management of Data (SIGMOD '95), pp. 92-103, 1995.

[7] A. Sistla, C. Yu, and R. Haddad, "Reasoning About Spatial Relationships in Picture Retrieval Systems," Proc. 20th Int'l Conf. Very Large Data Bases (VLDB '94), pp. 570-581, 1994.

[8] B. Bennett, A. Isli, and A. Cohn, "When Does a Composition Table Provide a Complete and Tractable Proof Procedure for a Relational Constraint Language," Proc. 15th Int'l Joint Conf. Artificial Intelligence (IJCAI '97) Workshop Spatial and Temporal Reasoning, 1997.

[9] K. Zimmermann, "Enhancing Qualitative Spatial ReasoningCombining Orientation and Distance," Proc. European Conf. Spatial Information Theory (COSIT '93), pp. 69-76, 1993.

[10] A. Abdelmoty and H. Williams, "Approaches to the Representation of Qualitative Spatial Relationships for Geographic Databases," Proc. Sixth Int'l GIS Workshop Advanced Geographic Data Modeling (AGDM '94), 1994.

[11] R. Billen and E. Clementini, "A Model for Ternary Projective Relations between Regions," Proc. Ninth Int'l Conf. Extending Database Technology (EDBT '04), pp. 310-328, 2004.

[12] C. Freksa, "Using Orientation Information for Qualitative Spatial Reasoning," Proc. Conf. Spatial Information Theory (COSIT '92), pp. 162-178, 1992.

[13] B. Faltings, "Qualitative Spatial Reasoning Using Algebraic Topology," Proc. Conf. Spatial Information Theory (COSIT '95), 1995.

[14] A. Mukerjee and G. Joe, "A Qualitative Model for Space," Proc. Eighth Nat'l Conf. Artificial Intelligence (AAAI '90), pp. 721-727, 1990.
[15] D. Papadias, "Relation-Based Representation of Spatial Knowledge," PhD dissertation, Dept. of Electrical and Computer Eng., Nat'1 Technical Univ. of Athens, 1994.

[16] G. Ligozat, "Reasoning about Cardinal Directions," J. Visual Languages and Computing, vol. 9, pp. 23-44, 1998.

[17] S. Skiadopoulos and M. Koubarakis, "Composing Cardinal Direction Relations," Artificial Intelligence, vol. 152, no. 2, pp. 143-171, 2004.

[18] R. Goyal, "Similarity Assessment for Cardinal Directions between Extended Spatial Objects," PhD dissertation, Dept. of Spatial Information Science and Eng., Univ. of Maine, Apr. 2000.

[19] S. Skiadopoulos and M. Koubarakis, "On the Consistency of Cardinal Directions Constraints," Artificial Intelligence, vol. 163, no. 1, pp. 91-135, 2005.

[20] J. Huttenlocher, L. Hedges, and S. Duncan, "Categories and Particulars: Prototype Effects in Estimating Spatial Location," Psychological Rev., vol. 98, no. 3, pp. 352-376, 1991.

[21] N. Franklin, L. Henkel, and T. Zangas, "Parsing Surrounding Space into Regions," Memory and Cognition, vol. 23, pp. 397-407, 1995.

[22] R. Hartley and A. Zisserman, Multiple View Geometry in Computer Vision, second ed. Cambridge Univ. Press, 2004.

[23] Wikipedia, "Field of View," http://en.wikipedia.org/wiki/ Field_of_view, 2006.

[24] D. Forsyth and J. Ponce, Computer Vision: A Modern Approach, first ed. Prentice Hall, 2002.

[25] J. Kuffner and J. Latombe, "Memory and Learning for Virtual Humans," Proc. IEEE Int'l Conf. Computer Animation (CA '99), pp. 118-127, 1999.

[26] D. Peuquet and Z. Ci-Xiang, "An Algorithm to Determine the Directional Relationship between Arbitrarily Shaped Polygons in the Plane," Pattern Recognition, vol. 20, no. 1, pp. 65-74, 1987.

[27] M. Grigni, D. Papadias, and C. Papadimitriou, "Topological Inference," Proc. Int'l Joint Conf. Artificial Intelligence (IJCAI '95), pp. 901-907, 1995.

[28] G. Ligozat, "When Tables Tell It All: Qualitative Spatial and Temporal Reasoning Based on Linear Ordering," Proc. Conf. Spatial Information Theory (COSIT '01), pp. 60-75, 2001.

[29] D. Randell, A. Cohn, and Z. Cui, "Computing Transitivity Tables: A Challenge for Automated Theorem Provers," Proc. 11th Int'l Conf. Automated Deduction (CADE '92), pp. 786-790, June 1992.

[30] M. Egenhofer and R. Franzosa, "Point Set Topological Relations," Int'l J. Geographical Information Systems, vol. 5, pp. 161-174, 1991.

[31] D. Papadias, N. Arkoumanis, and N. Karacapilidis, "On the Retrieval of Similar Configurations," Proc. Eighth Int'l Symp. Spatial Data Handling (SDH' '98), pp. 510-521, 1998.

[32] A. Tarski, “On the Calculus of Relations," J. Symbolic Logic, vol. 6, pp. 73-89, 1941.

[33] I. Düntsch, H. Wang, and S. McCloskey, "A Relation-Algebraic Approach to the Region Connection Calculus," Theoretical Computer Science, vol. 255, pp. 63-83, 2001.

[34] S. Li and M. Ying, "Region Connection Calculus: Its Models and Composition Table," Artificial Intelligence, vol. 145, pp. 121-146, 2003.

[35] B. Nebel and H.-J. Bürckert, "Reasoning about Temporal Relations: A Maximal Tractable Subclass of Allen's Interval Algebra," J. ACM, vol. 42, no. 1, pp. 43-66, 1995.

[36] S. Skiadopoulos, C. Giannoukos, N. Sarkas, P. Vassiliadis, T. Sellis, and M. Koubarakis, "Computing and Managing Cardinal Direction Relations," IEEE Trans. Knowledge and Data Eng., vol. 17, no. 12, pp. 1610-1623, Dec. 2005.

[37] T. Brinkhoff, H.-P. Kriegel, and R. Schneider, "Comparison of Approximations of Complex Objects Used for ApproximationBased Query Processing in Spatial Database Systems," Proc. Ninth IEEE Int'l Conf. Data Eng. (ICDE '93), pp. 40-49, 1993. 


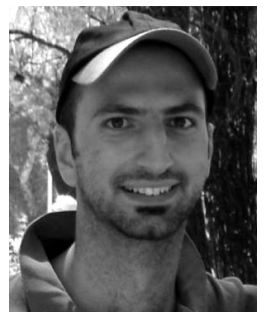

Spiros Skiadopoulos received the diploma and $\mathrm{PhD}$ degrees from the National Technical University of Athens (NTUA) and the MPhil from the University of Manchester Institute of Science and Technology (UMIST). He is currently an assistant professor in the Department of Computer Science and Technology, University of Peloponnese. He has published more than 25 papers in the areas of spatial and temporal databases, constraint databases and reasoning, query evaluation and optimization, and data warehouses.

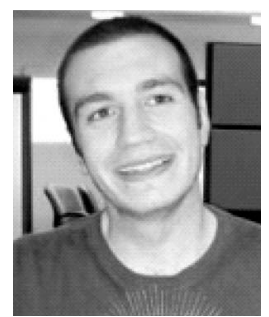

Nikos Sarkas received the diploma degree in electrical and computer engineering from the National Technical University of Athens (NTUA) in 2004 and is currently working toward the PhD degree at the University of Toronto. His research interests include spatial reasoning, top-k query evaluation, and Web search.

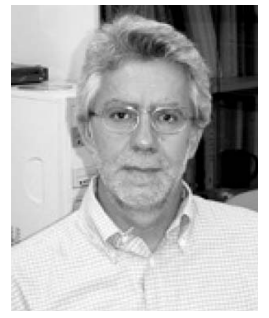

Timos Sellis received the diploma degree in electrical engineering in 1982 from the National Technical University of Athens (NTUA), the MSc degree in computer science from Harvard University in 1983, and the $\mathrm{PhD}$ degree in computer science from the University of California, Berkeley, in 1986, where he was a member of the INGRES group. In 1986, he joined the Department of Computer Science, University of Maryland, College Park, as an assistant professor and became an associate professor in 1992. Between 1992 and 1996, he was an associate professor in the Computer Science Division, NTUA, where he is currently a full professor. He is also the head of the Knowledge and Database Systems Laboratory at NTUA. He was an organizing chair of the 23rd International Conference on Very Large Data Bases (VLDB 1997) and the program committee (PC) chair of the 2001 ACM International Conference on Management of Data (SIGMOD). He was the president of the National Council for Research and Technology of Greece (2001-2003) and a member of the VLDB Endowment (1996-2000). His research interests include peer-to-peer database systems, data warehouses, the integration of Web and databases, and spatial database systems. He has published more than 120 papers in refereed journals and international conferences in the above areas and has been an invited speaker in major international events. He was a recipient of the prestigious Presidential Young Investigator (PYI) Award, given by the President of the United States of America to the most talented new researchers, in 1990 and of the VLDB 1997 10-Year Paper Award for his work on spatial databases. He is a member of the IEEE.

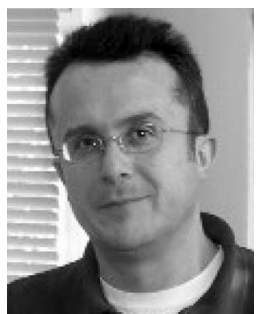

Manolis Koubarakis received the diploma (BSc) degree in mathematics from the University of Crete, the MSc degree in computer science from the University of Toronto, and the $\mathrm{PhD}$ degree in computer science from the National Technical University of Athens. He is an associate professor in the Department of Informatics and Telecommunications, National and Kapodistrian University of Athens. He has published more than 85 papers in the areas of database and knowledge-based systems, temporal and spatial reasoning, constraint programming, intelligent agents, semantic web, peer-topeer, and grid computing. His research has been funded by the European Commission, the Greek General Secretariat for Research and Technology, and industrial sources. He is a member of the IEEE Computer Society.

$\triangleright$ For more information on this or any other computing topic, please visit our Digital Library at www.computer.org/publications/dlib. 